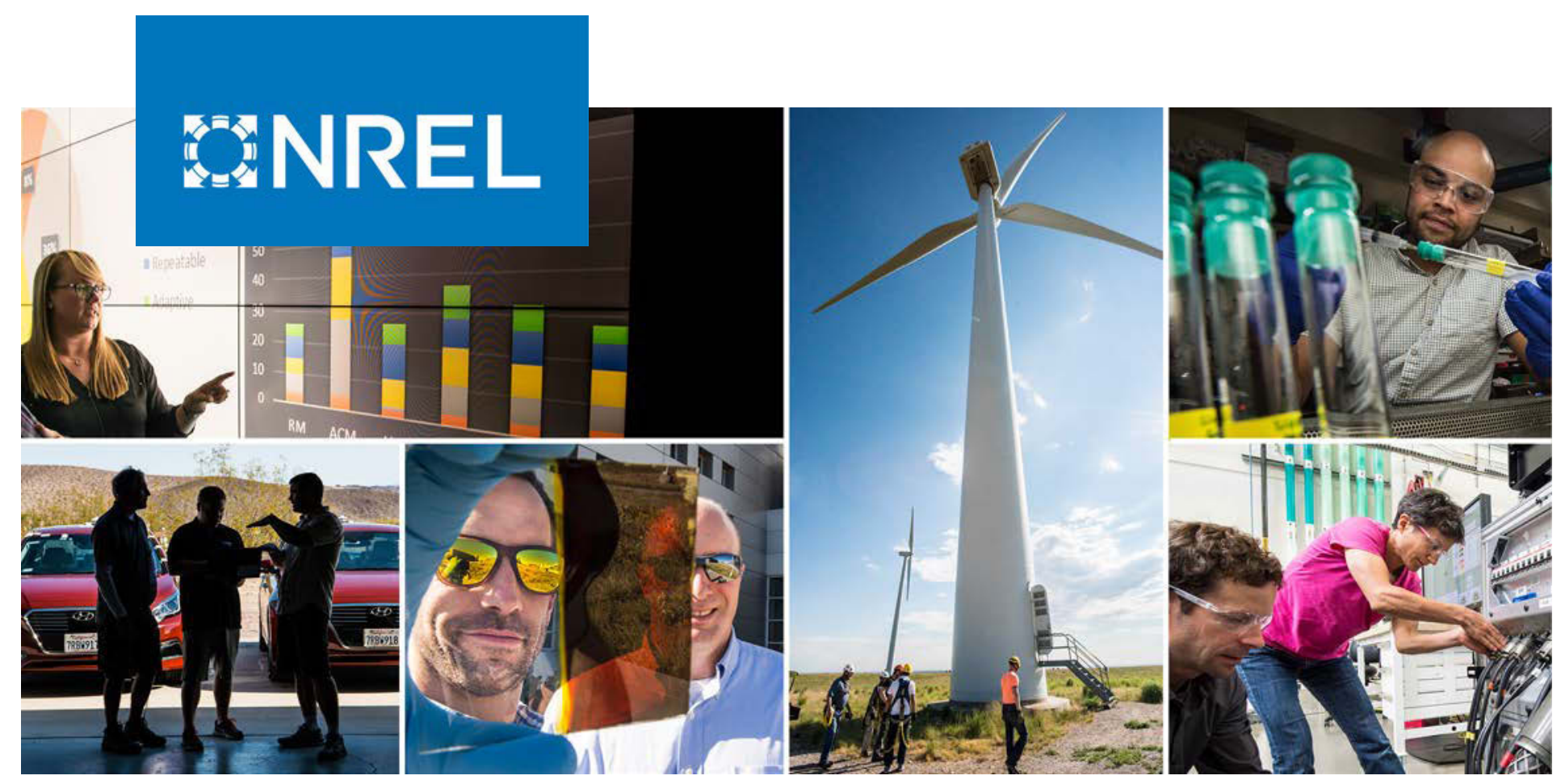

\title{
Ex Situ Catalytic Fast Pyrolysis of Lignocellulosic Biomass to Hydrocarbon Fuels: 2020 State of Technology
}

Abhijit Dutta, ${ }^{1}$ Calvin Mukarakate,${ }^{1}$ Kristiina lisa, ${ }^{1}$ Huamin Wang, ${ }^{2}$ Michael Talmadge, ${ }^{1}$ Daniel Santosa, ${ }^{2}$ Kylee Harris, ${ }^{1}$ Frederick Baddour, ${ }^{1}$ Damon Hartley, ${ }^{3}$ Hao Cai, ${ }^{4}$ Longwen Ou, ${ }^{4}$ Joshua Schaidle,${ }^{1}$ and Michael Griffin ${ }^{1}$

1 National Renewable Energy Laboratory

2 Pacific Northwest National Laboratory

3 Idaho National Laboratory

4 Argonne National Laboratory

NREL is a national laboratory of the U.S. Department of Energy Office of Energy Efficiency \& Renewable Energy

Operated by the Alliance for Sustainable Energy, LLC

This report is available at no cost from the National Renewable Energy Laboratory (NREL) at www.nrel.gov/publications.
Technical Report

NREL/TP-5100-80291

June 2021 


\section{GNREL}

\section{Ex Situ Catalytic Fast Pyrolysis of Lignocellulosic Biomass to Hydrocarbon Fuels: 2020 State of Technology}

Abhijit Dutta, ${ }^{1}$ Calvin Mukarakate, ${ }^{1}$ Kristiina lisa, ${ }^{1}$ Huamin Wang, ${ }^{2}$ Michael Talmadge,${ }^{1}$ Daniel Santosa, ${ }^{2}$ Kylee Harris, ${ }^{1}$ Frederick Baddour, ${ }^{1}$ Damon Hartley, ${ }^{3}$ Hao Cai, ${ }^{4}$ Longwen Ou, ${ }^{4}$ Joshua Schaidle, ${ }^{1}$ and Michael Griffin ${ }^{1}$

1 National Renewable Energy Laboratory

2 Pacific Northwest National Laboratory

3 Idaho National Laboratory

4 Argonne National Laboratory

\section{Suggested Citation}

Dutta, Abhijit, Calvin Mukarakate, Kristiina lisa, Huamin Wang, Michael Talmadge, Daniel Santosa, Kylee Harris, Frederick Baddour, et al. 2021. Ex Situ Catalytic Fast Pyrolysis of Lignocellulosic Biomass to Hydrocarbon Fuels: 2020 State of Technology. Golden, CO: National Renewable Energy Laboratory. NREL/TP-5100-80291.

https://www.nrel.gov/docs/fy21osti/80291.pdf.

NREL is a national laboratory of the U.S. Department of Energy Office of Energy Efficiency \& Renewable Energy Operated by the Alliance for Sustainable Energy, LLC

This report is available at no cost from the National Renewable Energy Laboratory (NREL) at www.nrel.gov/publications.

Contract No. DE-AC36-08GO28308
Technical Report

NREL/TP-5100-80291

June 2021

National Renewable Energy Laboratory 15013 Denver West Parkway Golden, CO 80401

303-275-3000 • www.nrel.gov 


\section{NOTICE}

This work was authored in part by the National Renewable Energy Laboratory, operated by Alliance for Sustainable Energy, LLC, for the U.S. Department of Energy (DOE) under Contract No. DE-AC36-08GO28308; Idaho National Laboratory, operated for DOE by Battelle Energy Alliance under contract DE-AC07-05ID14517; and Argonne National Laboratory, managed by UChicago Argonne, LLC, under DOE Contract No. DE-AC02-06CH11357. Funding provided by U.S. Department of Energy Office of Energy Efficiency and Renewable Energy Bioenergy Technologies Office. The views expressed herein do not necessarily represent the views of the DOE or the U.S. Government.

This report is available at no cost from the National Renewable Energy Laboratory (NREL) at www.nrel.gov/publications.

U.S. Department of Energy (DOE) reports produced after 1991 and a growing number of pre-1991 documents are available free via www.OSTI.gov.

Cover Photos by Dennis Schroeder: (clockwise, left to right) NREL 51934, NREL 45897, NREL 42160, NREL 45891, NREL 48097, NREL 46526.

NREL prints on paper that contains recycled content. 


\section{Acknowledgments}

The authors wish to thank the following researchers for their contributions to this work: Kellene McKinney, Scott Palmer, Carson Pierce, Andy Larson, Sean West, Jon Luecke, Cameron Hays, Earl Christensen, Andrew Young, Braden Peterson, Kim Magrini, Kurt Van Allsburg, Daniel Carpenter, Matt Yung, Nolan Wilson, Mark Nimlos, and Zia Abdullah from the National Renewable Energy Laboratory (NREL); Michael Thorson from Pacific Northwest National Laboratory; Jordan Klinger from Idaho National Laboratory; and Bruce Adkins from Oak Ridge National Laboratory. We appreciate communications support from Michael Deneen and Kathy Cisar from NREL. We thank Mike Watson and Luke Tuxworth from Johnson Matthey for their cooperative work with NREL on catalyst research (under a cooperative research and development agreement). We also thank Andrea Bailey and Trevor Smith from the Bioenergy Technologies Office for their support and guidance.

This effort is supported by many individuals across multiple national laboratories. The authors apologize in advance for inadvertent omissions in acknowledging any contributors to this project. 


\section{List of Acronyms}

2FBR

ANL

Btu

CFP

FCI

FY

GGE

GHG

INL

ISBL

LHV

MEK

MFSP

MM

NREL

PNNL

SCSA

SOT

TEA

TIC

TPEC 2-inch fluidized-bed reactor

Argonne National Laboratory

British thermal unit

catalytic fast pyrolysis

fixed capital investment

fiscal year

gallon gasoline equivalent

greenhouse gas

Idaho National Laboratory

inside battery limits

lower heating value

methyl-ethyl-ketone

minimum fuel selling price

million

National Renewable Energy Laboratory

Pacific Northwest National Laboratory

supply chain sustainability analysis

state of technology

techno-economic analysis

total installed cost

total purchased equipment cost 


\section{Executive Summary}

This report documents the progress in research funded by the U.S. Department of Energy's Office of Energy Efficiency and Renewable Energy Bioenergy Technologies Office for the conversion of biomass to infrastructure-compatible liquid hydrocarbon fuels via catalytic fast pyrolysis (CFP). The focus of this report is on research learnings since our 2019 state of technology (SOT) publication ${ }^{1}$ and corresponding improvements in assessed model costs and life cycle impacts. Upon scaling our 2020 SOT bench-scale experimental performance to a modeled 2,000-dry-tons-per-day woody biomass conceptual process, we were able to achieve our 2022 target metrics of $<\$ 3$ per gasoline gallon equivalent (GGE) modeled minimum fuel selling price (MFSP) for liquid hydrocarbon fuel blendstock production, along with $>60 \%$ greenhouse gas (GHG) reduction compared to petroleum-derived gasoline. Specifically, our base case modeled MFSP was $\$ 2.83 / \mathrm{GGE}$ with a $78 \%$ GHG reduction; the MFSP range upon considering some key uncertainties in the current analysis was \$2.68-\$2.99/GGE. Key advances in fiscal year (FY) 2020 include (1) improvements in understanding the potential to leverage existing refinery infrastructure, (2) more experimental proof regarding our previously proposed concept of including chemical coproducts along with primary fuels production, and (3) high overall carbon utilization with the combined slate of fuels and coproducts. Future research needs include further study of hydrogen utilization at scale, full demonstration/integration of light oxygenate capture and purification, and extended-duration operations for both catalytic fast pyrolysis and refinery coprocessing. Details of our conceptual process configuration are discussed in the text, including fixed-bed ex situ CFP, recovery of light oxygenated coproducts, and co-hydrotreating of the CFP oil at a petroleum refinery. Sensitivity cases are included to show modeled performance summaries for two key alternate configuration scenarios in which: (1) no light oxygenated coproducts are recovered, or (2) the CFP oil is sent to a stand-alone hydrotreating/hydrocracking operation at the biorefinery instead of being co-hydrotreated at a petroleum refinery. Further details of research achievements are expected to be published/presented in the near future, along with a closeout report signifying a potential transition of this conversion pathway toward further commercial relevance after addressing identified risks. Relevant sections from previous SOT and design reports are repeated in some portions of the text to help provide background and present information in a consistent and comparable format with previous reports. Interested readers and those unfamiliar with biomass CFP are provided with more detailed references for additional background.

\section{Background Information}

In the ex situ CFP pathway, biomass undergoes rapid deconstruction in a fast pyrolysis reactor at approximately $500^{\circ} \mathrm{C}\left(932^{\circ} \mathrm{F}\right)$, followed by the separation of produced solids (char and mineral matter) from vapors (including permanent gases); the vapors are then sent to an ex situ catalytic reactor for upgrading. Upgrading involves deoxygenation, hydrogenation, and carbon-carbon coupling, and this renders the vapors significantly less reactive and more amenable to further processing after condensation (the condensation product is known as CFP oil). Solids removal prior to the ex situ upgrading step provides an advantage with respect to catalyst stability and choices (relative to in situ upgrading, in which the catalyst mixes with biomass-derived solids within the fast pyrolysis reactor); catalyst choices can be further broadened to include supported noble metals in fixed-bed systems. The effectiveness of this ex situ vapor upgrading step for CFP

\footnotetext{
${ }^{1}$ Available at: https://www.nrel.gov/docs/fy20osti/76269.pdf.
} 
oil quality improvement has been verified, with experiments proving that single-step hydrotreating can deoxygenate the liquid product to less than $1 \mathrm{wt} \%$ oxygen. Catalyst stability during hydrotreating of non-catalytic fast pyrolysis bio-oil is a major challenge; multiple hydrotreating steps are required unless the catalytic vapor upgrading step is included.

Initial research efforts associated with this project (beginning in 2014) were focused on zeolite catalysts in a fluidized CFP reactor system; this was detailed in a 2015 design report. ${ }^{2}$ The 2017 SOT showed significant liquid-range product yield improvements using a $\mathrm{Pt} / \mathrm{TiO}_{2}$ catalyst in the presence of added hydrogen, which led to the choice of a fixed-bed ex situ configuration for further research improvements from 2017 to 2020, including this current SOT assessment.

\section{Research and Development Since the 2019 SOT Report}

\section{Experimental Equipment Modifications}

After the 2019 SOT experiments, the bench-scale ex situ CFP reactor system (2-inch fluidizedbed reactor [2FBR] system at $\mathrm{NREL}^{3}$ ) was completely rebuilt and recommissioned to include enhanced safety features and allow operational efficiency improvements. The 2019 SOT experiments were repeated to ensure that we obtained comparable yields, and our experiments reproduced previous quantitative benchmarks, including near-100\% carbon balance closures. As in previous years, hydrotreating experiments were conducted at Pacific Northwest National Laboratory (PNNL) and the National Renewable Energy Laboratory (NREL). Continuous hydrotreating experimental setups were used at both PNNL and NREL (versus a batch system used at NREL in previous years). The light oxygenates adsorption/desorption system, based on model compound tests in FY 2019, was designed and deployed to capture light oxygenates from the CFP vapor stream generated by the $2 \mathrm{FBR}$.

\section{Key Advancements in FY 2020 Toward Addressing Previous Uncertainties}

\section{CFP Oil Co-Hydrotreating}

The FY 2019 SOT included assumptions of co-hydrotreating performance based on preliminary experiments. Co-hydrotreating experiments at PNNL and NREL in FY 2020 helped solidify our understanding of yields and performance for co-hydrotreating CFP oil with straight-run diesel. The results reported here and used in the techno-economic analysis (TEA) are from cohydrotreating experiments with CFP oil and straight-run diesel at a 10:90 vol \% feed ratio. The experimental results showed 95\%-97\% carbon efficiency for conversion of biogenic compounds in CFP oil to liquid fuel blendstocks. We lowered the efficiency assumption to $94.5 \%$ in the TEA base case to allow for uncertainties and potential additional unaccounted losses in industrial implementations. The oxygen content of the coprocessed fuel product was below detection limits, and the cetane numbers of the diesel-range cuts were 50-55. These co-hydrotreating runs were conducted at $1,200 \mathrm{psi}$ and $340^{\circ} \mathrm{C}$ using a NiMo/alumina catalyst at a weight hourly space velocity of $1 \mathrm{~h}^{-1}$ and time on stream of at least 50 hours, both at PNNL and NREL. For the tests

\footnotetext{
${ }^{2}$ Available at: https://www.nrel.gov/docs/fy15osti/62455.pdf.

${ }^{3}$ Griffin, M. B. et al. 2018. "Driving towards cost-competitive biofuels through catalytic fast pyrolysis by rethinking catalyst selection and reactor configuration." Energy \& Environmental Science 11: 2904-2918.

http://dx.doi.org/10.1039/C8EE01872C.
} 
reported here, no obvious adverse effects of the CFP oil co-feed were observed on the hydrodesulfurization of straight-run diesel, compared to the runs with straight-run diesel only. However, the impact of CFP oil co-feed on the hydrotreating catalyst, reactor, products, and downstream processes, especially during longer-term operations, needs further investigation and is the subject of extensive research efforts.

\section{Recovery of Light Oxygenates}

The FY 2019 SOT TEA was based on adsorption/desorption performance using model compound mixtures of the predominant light oxygenates with key permanent gases, as measured in the uncondensed exhaust from the 2FBR system. Although acetaldehyde, acetone, and 2butanone were the predominant oxygenated compounds ( $>75 \mathrm{wt} \%)$, only acetone and 2butanone (also known as methyl-ethyl-ketone [MEK]) were targeted for recovery and purification as saleable products because of their compatibility with current market sizes. In FY 2020, further experiments showed that the adsorption/desorption strategy for the capture of light oxygenates, which worked with model compounds in FY 2019, also works with real CFP vapors. Although no problems were encountered in the two to three cycles of operation, we understand that additional testing will be necessary to prove long-term robustness of the system. Near- $100 \%$ recovery of acetone and 2-butanone was achieved during the adsorption/desorption operations using silica gel as the adsorbent, thus resolving one of the key uncertainties related to the ability of the system to selectively adsorb light oxygenates from the complex gas/vapor stream. The next challenge after the recovery of a mixed stream of acetone, 2-butanone, and other compounds via desorption was achieving chemical-grade purity of desired saleable products. An extensive phase-equilibrium-based separations model (including several distillation columns and liquid-liquid separations) was developed in Aspen Plus for the purification of acetone and 2butanone using the detailed experimental speciation of the desorbed stream. The UNIFACDortmund $^{4}$ property method was used as the basis for phase equilibrium behavior. The complexities of obtaining high-purity products, associated losses, energy consumption, and the use of solvents to favorably affect phase equilibrium were captured in the model. This is a standard approach for the modeling and design of chemical purification systems. Although the UNIFAC model predicted multiple azeotropes, thus driving up separations complexity and costs, the biggest related uncertainty is whether the UNIFAC model reasonably predicts the phase behavior and azeotropes. In general, comparison of sampled binary systems behavior between UNIFAC and experimentally fitted models shows reasonable parity, thus lending credence that our modeling adequately captures the losses, achievable purities, and costs. The modeled purities of acetone and 2-butanone were 97.7 and $99.6 \mathrm{wt} \%$, respectively, with $70 \%$ and 39\% recovery rates of the purified coproducts, respectively. The recovery rates can likely be optimized by changing the condensation and recovery configuration, but we decided to leave a conservative coproduct credit estimate at this time, pending additional experiments to prove long-term robustness and property method validation; additional recovery of these saleable coproducts can significantly benefit the economics and further lower the MFSP. The estimated capital cost for the light oxygenates recovery and purification system is included in the report. If resources permit, we will conduct phase equilibrium measurements for further validation of the UNIFAC predictions, along with additional adsorption/desorption experiments to prove robustness of our

\footnotetext{
${ }^{4}$ Gmehling, J. et al. 2002. “A Modified UNIFAC (Dortmund) Model. 4. Revision and Extension.” Industrial \& Engineering Chemistry Research 41 (6): 1678-1688. https://doi.org/10.1021/ie0108043.
} 
approach toward producing saleable high-purity acetone and 2-butanone from this process. We used 5-year average prices for both acetone ${ }^{5}$ and 2-butanone, ${ }^{6}$ with coproduct values of $\$ 0.442 / \mathrm{lb}$ and $\$ 0.771 / \mathrm{lb}$, respectively.

\section{Life Cycle GHG Emissions}

Life cycle analysis by Argonne National Laboratory for the FY 2020 SOT base case shows a $78 \%$ GHG reduction over petroleum-derived gasoline. This is even after accounting for a large GHG footprint from the use of natural gas for co-hydroprocessing at the petroleum refinery. The primary reasons for the favorable overall GHG emissions, despite the assumed use of natural gas for hydrogen production at the petroleum refinery, are the displacement credits from acetone, 2butanone, and electricity coproducts, which more than offset the impacts of natural gas use.

\section{Sensitivity Cases for Key Uncertainties Around the FY 2020 SOT Base Case}

1. The assumption of a CFP oil co-hydrotreating carbon efficiency of $96.5 \%$ vs. the base case model assumption of $94.5 \%$ reduced the modeled MFSP from $\$ 2.83 / \mathrm{GGE}$ to $\$ 2.78 / \mathrm{GGE}$; this covers impact of the experimentally reported $95 \%-97 \%$ carbon efficiency range.

2. Increasing feedstock cost from $\$ 67.03 /$ dry U.S. ton to $\$ 70.15 /$ dry U.S. ton (used in the FY 2019 SOT) increased the MFSP from \$2.83/GGE to \$2.88/GGE; this reflects the potential impact of additional ash removal requirements during feedstock preprocessing.

3. A $20 \%$ increase in value from coproducts (e.g., via enhanced recovery) reduced the MFSP from $\$ 2.83 / \mathrm{GGE}$ to $\$ 2.72 / \mathrm{GGE}$, whereas a $20 \%$ decrease in the value from coproducts increased the MFSP from $\$ 2.83 / \mathrm{GGE}$ to $\$ 2.94 / \mathrm{GGE}$.

Overall, combining the optimistic and pessimistic cases mentioned above, we arrived at an MFSP range of $\$ 2.68-\$ 2.99 /$ GGE around the base case MFSP of $\$ 2.83 /$ GGE.

\footnotetext{
${ }^{5}$ Pampell, M. and T. Spyra. 2018. Chemical Economics Handbook: Acetone. London, UK: IHS Markit. ${ }^{6}$ Zhang, E., A. Bland, E. Greiner, and T. Kumamoto. 2018. Chemical Economics Handbook: Methyl Ethyl Ketone $(M E K)$. London, UK: IHS Markit.
} 


\section{Key Results}

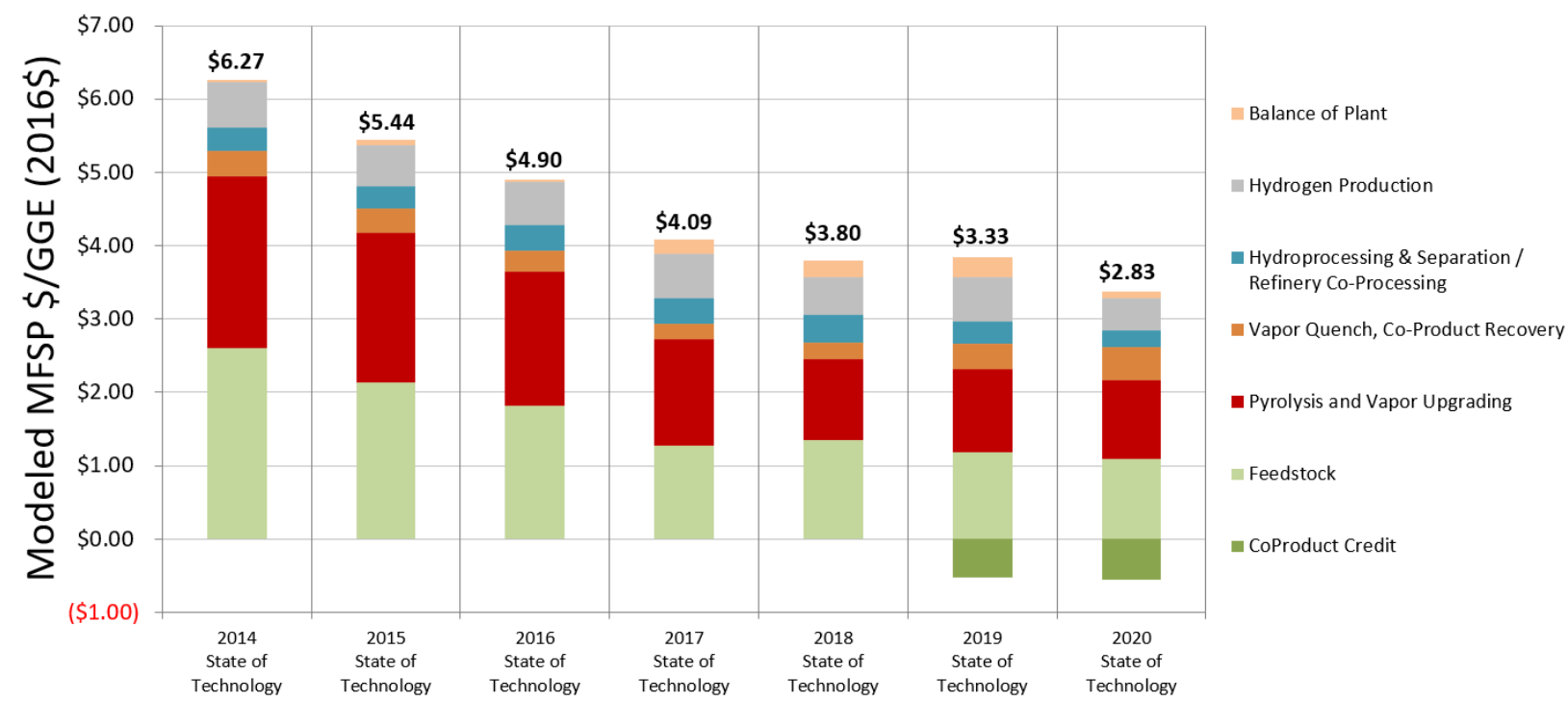

Figure ES-1. Modeled MFSP for 2014-2020 SOTs. There was a transition from CFP using zeolite catalysts in a fluidized bed (2014-2016) to a Pt/TiO ${ }_{2}$ catalyst in a fixed bed (2017-2020). Acetone and 2-butanone coproducts were included in 2019 and 2020. 
Table ES-1. Key Metrics in TEA Model for the 2020 Target and SOT Cases

\begin{tabular}{|c|c|c|}
\hline & FY 2020 Target $^{a}$ & FY 2020 SOT \\
\hline \multicolumn{3}{|l|}{ Fast Pyrolysis Intermediate ${ }^{b}$} \\
\hline Gas Species-CO, $\mathrm{CO}_{2}, \mathrm{C}_{1}-\mathrm{C}_{4}$ (wt \% of dry biomass) & 13 & 13 \\
\hline Organics (wt \% of dry biomass) & 64 & 64 \\
\hline Water (wt \% of dry biomass) & 11 & 11 \\
\hline Char (wt \% of dry biomass) & 12 & $11^{\mathrm{b}}$ \\
\hline Fixed Bed-Online: Regen Reactors ${ }^{c}$ & $2: 2$ & $2: 2$ \\
\hline Gas, includes condensables (wt \% of dry biomass) & 38 & 41.8 \\
\hline Aqueous phase (wt \% of dry biomass) & 24 & 20.5 \\
\hline Carbon loss (\% of $\mathrm{C}$ in biomass) & 4.4 & 1.8 \\
\hline Organic phase (wt \% of dry biomass) & 23 & 24 \\
\hline H/C molar ratio & 1.2 & 1.3 \\
\hline Oxygen (wt \% in organic phase) & 15 & 16.6 \\
\hline Carbon efficiency (\%) & $35^{d}$ & $35.7^{d}$ \\
\hline Solid losses, char + coke (wt $\%$ of dry biomass) & $12+2$ & $11.1+1.7$ \\
\hline \multicolumn{3}{|l|}{ Final Fuel Blendstock } \\
\hline Yield (\%, w/w dry biomass) & 19 & 19.6 \\
\hline Hydroprocessing carbon efficiency $(\%)^{c}$ & 93.5 e & 94.5 e \\
\hline Overall carbon efficiency to fuel (\% of C in biomass) & 33 & 33.7 \\
\hline $\begin{array}{l}\text { Overall carbon efficiency to fuel (\% of C in biomass + } \\
\text { natural gas) }\end{array}$ & 33 & 33.7 \\
\hline Total product (GGE/dry U.S. ton) & 59 & 61 \\
\hline Gasoline-range product (gallons/dry U.S. ton) & 32 & 31.7 \\
\hline Diesel-range product (gallons/dry U.S. ton) & 26 & 27.9 \\
\hline Gasoline/diesel-range product (\% GGE basis) & $52 / 48$ & $50 / 50$ \\
\hline Oxygen content in cumulative product (wt \%) & 0.5 & $0.5^{f}$ \\
\hline Acetone + MEK coproduct credit (\$/GGE) & 0.52 & 0.55 \\
\hline Minimum fuel selling price (\$/GGE) & 3.09 & 2.83 \\
\hline \multicolumn{3}{|l|}{ Natural Gas ${ }^{\mathrm{g}}$ and Electricity } \\
\hline Natural gas at biorefinery ( $\%$ of biomass, LHV ${ }^{\mathrm{h}}$ basis) & 0.5 & 0.1 \\
\hline Natural gas at petroleum refinery (\% biomass, LHV) & 16 & 18 \\
\hline Surplus electricity at biorefinery (\$/GGE) & 0.21 & 0.27 \\
\hline \multicolumn{3}{|l|}{ Fuel Blendstock Production Efficiency } \\
\hline Biomass feedstock (\%, LHV basis) & 43.6 & 45.1 \\
\hline
\end{tabular}

a From https://www.nrel.gov/docs/fy20osti/76269.pdf. ${ }^{b}$ Fast pyrolysis intermediate yields maintained from 2015 design report (https://www.nrel.gov/docs/fy15osti/62455.pdf). Lower experimental char yield in FY 2020 was used in the SOT TEA, and the other yields were prorated to a $100 \%$ total (values shown are rounded off). ${ }^{\mathrm{c}}$ Model separation and distribution may differ slightly from experiments because of differences in configurations and other assumptions. Parameters with greatest economic impacts are matched closely. Most of the deviations lead to more conservative (higher) MFSPs. ${ }^{d}$ Additional carbon in condensables not shown here, but included in Appendix A. e Cohydroprocessing. ${ }^{\mathrm{f}}$ Oxygen was not detected in co-hydrotreated product mixture. ${ }^{9}$ Small amount of natural gas at biorefinery, but significant quantity used at petroleum refinery. ${ }^{\mathrm{h}}$ Lower heating value. 
Table ES-2. Economic Summary (Modeled) for the 2020 SOT with Co-Hydroprocessing Option

Process Engineering Analysis for Hydrocarbon Fuel Production

via Ex Situ Upgrading of Fast Pyrolysis Vapors

Potential Research-Driven Pathway for Cost-Competitiveness by 2022

2,000 Dry Metric Tonnes Biomass per Day

All Values in $2016 \$$

Minimum Fuel Selling Price (MFSP)

Gasoline Blendstock Production

Diesel Blendstock Production Total Gasoline Equivalent Production Catalytic Fast Pyrolysis Oil

Light Oxygenated Coproducts

Delivered Feedstock Cost Internal Rate of Return (After-Tax) On-Stream Factor

Capital Costs

100: Feedstock (Additional Dryer \& Blower Only)

200: Fast Pyrolysis \& Vapor Upgrading

300: Vapor Quench \& CFP Co-Products

400: Hydroprocessing \& Separation

500: Hydrogen Plant

600: Steam System \& Power Generation

700: Cooling Water \& Other Utilities

800: Water Management

Total Installed Equipment Cost (TIC)

Land (115 Acres at \$14000 per Acre)

Site Development

(\% of ISBL)

Indirect Costs \& Project Contingency

(\% of TIC)

Fixed Capital Investment (FCl)

Working Capital

000

$\$ 25,900,000$

$\$ 543,820,000$

Loan Interest Rate

Loan Term (Years)

$8.0 \%$

10

Fixed Capital Investment per Annual GGE

$\$ 6.61$

$\$ 11.64$

Plant Operating Hours per Year

7884

On-Stream Percentage

$90.0 \%$

Maximum Yield Based on HHV of Feedstock + Natural Gas Theoretical GGE Production (MM GGE / Year)

Theoretical Yield (GGE / Dry Ton)

Current Yield (Actual / Theoretical)

102.9

142.1

$43.2 \%$

Overall Plant Efficiency - HHV \%

Overall Plant Efficiency - LHV \%

$44.7 \%$

$45.0 \%$
Total Capital Investment (TCI)

Total Installed Equipment Cost per Annual GGE

\$2.74 /Gallon Gasoline Blendstock

\$3.12 /Gallon Diesel Blendstock

\$2.83 /Gallon Gasoline Equivalent (GGE)

23.0 MM Gal per Year

20.2 MM Gal per Year

31.7 Gal per Dry US Ton Feedstock

44.5 MM GGE per Year

$0.94 \mathrm{MM}$ bbl per Year

27.9 Gal per Dry US Ton Feedstock

49.6 MM lb per year 61.4 GGE per Dry US Ton Feedstock

$1.3 \mathrm{bbl}$ per Dry US Ton Feedstock

$3.4 \% \mathrm{w} / \mathrm{w}$ of Dry Biomass

\% Acetone/Dry Biomass $\quad 0.5 \%$ MEK/Dry Biomass

$\$ 67.03$ per Dry U.S. Ton (Includes Capital Up to Throat of Pyrolyzer)

$10.0 \%$ Equity, \% of Total Investment $40.0 \%$

90.0\% CFP Oil (\$/bbl GE Product) $\$ 109.51$

\begin{tabular}{lr}
\multicolumn{2}{c}{ Operating Costs (c/ GGE Product) } \\
\hline Feedstock & 108.2 \\
Natural Gas & 0.2 \\
Catalysts & 9.1 \\
Sand & 0.6 \\
Other Raw Materials & 3.9 \\
Waste Disposal & 3.2 \\
Purchased Electricity & - \\
Fixed Costs & 57.7 \\
Refinery Coprocessing Cost & 22.6 \\
Chemical Coproduct Credit & $(54.7)$ \\
Electricity Coproduct Credit & $(27.4)$ \\
Capital Depreciation & 58.2 \\
Average Income Tax & 12.4 \\
Average Return on Investment & 89.2
\end{tabular}

\begin{tabular}{lr}
\multicolumn{2}{c}{ Operating Costs (\$ / Year) } \\
\hline Feedstock & $\$ 48,170,000$ \\
Natural Gas & $\$ 100,000$ \\
Catalysts & $\$ 4,050,000$ \\
Sand & $\$ 300,000$ \\
Other Raw Materials & $\$ 660,000$ \\
Waste Disposal & $\$ 1,400,000$ \\
Purchased Electricity & $\$ 0$ \\
Fixed Costs & $\$ 25,690,000$ \\
Refinery Coprocessing Cost & $\$ 10,070,000$ \\
Chemical Coproduct Credit & $-\$ 24,330,000$ \\
Electricity Coproduct Credit & $-\$ 12,180,000$ \\
Capital Depreciation & $\$ 25,900,000$ \\
Average Income Tax & $\$ 5,540,000$ \\
Average Return on Investment & $\$ 39,710,000$ \\
& \\
Total Plant Electricity Usage (kW) & 33,344 \\
$\quad$ Electricity Produced on Site (kW) & 59,751 \\
$\quad$ Electricity Purchased from Grid (kW) & 0 \\
$\quad$ Electricity Sold to Grid (kW) & 26,407
\end{tabular}

Plant Electricity Use (kWh /GGE) 5.91

Version:

PyVPU-2020-V096-HT_coproc-LO-R008-copied data

\begin{tabular}{llr} 
& Specific Operating Conditions \\
\hline Feed Rate & Dry Tonnes / Day & 2,000 \\
& Dry Tons / Day & 2,205 \\
Feedstock Cost & \$/Dry Ton & $\$ 67.03$ \\
& \$/Moisture+Ash Free Ton & $\$ 68.22$
\end{tabular}




\section{Table ES-3. Economic Summary (Modeled) for a 2020 Sensitivity Case without 2-Butanone and Acetone Coproducts}

\section{Process Engineering Analysis for Hydrocarbon Fuel Production via Ex Situ Upgrading of Fast Pyrolysis Vapors}

Potential Research-Driven Pathway for Cost-Competitiveness by 2022 2,000 Dry Metric Tonnes Biomass per Day All Values in $2016 \$$

Minimum Fuel Selling Price (MFSP)

Gasoline Blendstock Production Diesel Blendstock Production Total Gasoline Equivalent Production Catalytic Fast Pyrolysis Oil Light Oxygenated Coproducts

Delivered Feedstock Cost Internal Rate of Return (After-Tax) On-Stream Factor

Capital Costs

100: Feedstock (Additional Dryer \& Blower Only)

200: Fast Pyrolysis \& Vapor Upgrading 300: Vapor Quench \& CFP Co-Products 400: Hydroprocessing \& Separation 500: Hydrogen Plant

600: Steam System \& Power Generation 700: Cooling Water \& Other Utilities 800: Water Management

Total Installed Equipment Cost (TIC)

Land (115 Acres at \$14000 per Acre)

Site Development

(\% of ISBL)

Indirect Costs \& Project Contingency

(\% of TIC)

Fixed Capital Investment (FCl)

Working Capital

Total Capital Investment (TCl)

$\$ 25,280,000$

$\$ 530,800,000$

Loan Interest Rate

Loan Term (Years)

$8.0 \%$

Total Installed Equipment Cost per Annual GGE

Fixed Capital Investment per Annual GGE

$\$ 11.30$

Plant Operating Hours per Year

7884

On-Stream Percentage

$90.0 \%$

Maximum Yield Based on HHV of Feedstock + Natural Gas

Theoretical GGE Production (MM GGE / Year) 102.5

Theoretical Yield (GGE / Dry Ton) 141.5

Current Yield (Actual / Theoretical) $\quad 43.7 \%$

Overall Plant Efficiency - HHV \% $\quad 45.0 \%$

Overall Plant Efficiency - LHV \% $\quad 45.3 \%$

Version:

PyVPU-2020-V096-HT_coproc-LO-Electricity-Only-R008-copied data

\$3.11 /Gallon Gasoline Blendstock

\$3.54 /Gallon Diesel Blendstock

\$3.21 /Gallon Gasoline Equivalent (GGE)

$23.8 \mathrm{MM}$ Gal per Year

32.9 Gal per Dry US Ton Feedstock 19.6 MM Gal per Year $\quad 27.0 \mathrm{Gal}$ per Dry US Ton Feedstock 44.7 MM GGE per Year $\quad 61.8$ GGE per Dry US Ton Feedstock $0.95 \mathrm{MM}$ bbl per Year $\quad 1.3 \mathrm{bbl}$ per Dry US Ton Feedstock

$0.0 \mathrm{MM}$ lb per year $\quad 0.0 \% \mathrm{w} / \mathrm{w}$ of Dry Biomass

$0.0 \%$ Acetone/Dry Biomass $\quad 0.0 \%$ MEK/Dry Biomass $\$ 67.03$ per Dry U.S. Ton (Includes Capital Up to Throat of Pyrolyzer) $10.0 \%$ Equity, \% of Total Investment $40.0 \%$ 90.0\% CFP Oil (\$/bbl GE Product) $\$ 125.38$

\begin{tabular}{lr}
\multicolumn{2}{c}{ Operating Costs (c/ GGE Product) } \\
\hline Feedstock & 107.7 \\
Natural Gas & 0.1 \\
Catalysts & 9.0 \\
Sand & 0.6 \\
Other Raw Materials & 1.4 \\
Waste Disposal & 3.3 \\
Purchased Electricity & - \\
Fixed Costs & 56.4 \\
Refinery Coprocessing Cost & 22.4 \\
Chemical Coproduct Credit & - \\
Electricity Coproduct Credit & $(35.4)$ \\
Capital Depreciation & 56.5 \\
Average Income Tax & 12.2 \\
Average Return on Investment & 86.7
\end{tabular}

\begin{tabular}{|c|c|c|}
\hline \multicolumn{3}{|c|}{ Operating Costs (\$ / Year) } \\
\hline Feedstock & & $\$ 48,170,000$ \\
\hline \multicolumn{2}{|l|}{ Natural Gas } & $\$ 100,000$ \\
\hline \multicolumn{2}{|l|}{ Catalysts } & $\$ 4,030,000$ \\
\hline \multicolumn{2}{|l|}{ Sand } & $\$ 300,000$ \\
\hline \multicolumn{2}{|c|}{ Other Raw Materials } & $\$ 630,000$ \\
\hline \multicolumn{2}{|c|}{ Waste Disposal } & $\$ 1,460,000$ \\
\hline \multicolumn{2}{|c|}{ Purchased Electricity } & $\$ 0$ \\
\hline \multicolumn{2}{|c|}{ Fixed Costs } & $\$ 25,230,000$ \\
\hline \multicolumn{2}{|c|}{ Refinery Coprocessing Cost } & $\$ 10,010,000$ \\
\hline \multicolumn{2}{|c|}{ Chemical Coproduct Credit } & $\$ 0$ \\
\hline \multicolumn{2}{|c|}{ Electricity Coproduct Credit } & $-\$ 15,820,000$ \\
\hline \multicolumn{2}{|c|}{ Capital Depreciation } & $\$ 25,280,000$ \\
\hline \multicolumn{2}{|c|}{ Average Income Tax } & $\$ 5,440,000$ \\
\hline \multicolumn{2}{|c|}{ Average Return on Investment } & $\$ 38,790,000$ \\
\hline \multicolumn{2}{|c|}{ Total Plant Electricity Usage (kW) } & 31,938 \\
\hline \multicolumn{2}{|c|}{ Electricity Produced on Site (kW) } & 66,223 \\
\hline \multicolumn{2}{|c|}{ Electricity Purchased from Grid (kW) } & 0 \\
\hline \multicolumn{2}{|c|}{ Electricity Sold to Grid (kW) } & 34,284 \\
\hline \multicolumn{2}{|c|}{ Plant Electricity Use (kWh /GGE) } & 5.63 \\
\hline \multicolumn{3}{|c|}{ Specific Operating Conditions } \\
\hline \multirow[t]{2}{*}{ Feed Rate } & Dry Tonnes / Day & 2,000 \\
\hline & Dry Tons / Day & 2,205 \\
\hline \multirow[t]{2}{*}{ Feedstock Cost } & \$/Dry Ton & $\$ 67.03$ \\
\hline & \$/Moisture+Ash Free Ton & $\$ 68.22$ \\
\hline
\end{tabular}




\section{Table ES-4. Economic Summary (Modeled) for a 2020 Sensitivity Case with Hydroprocessing at}

the Biorefinery (i.e., no co-hydroprocessing)

\section{Process Engineering Analysis for Hydrocarbon Fuel Production via Ex Situ Upgrading of Fast Pyrolysis Vapors}

Potential Research-Driven Pathway for Cost-Competitiveness by 2022 2,000 Dry Metric Tonnes Biomass per Day

All Values in 2016\$

Minimum Fuel Selling Price (MFSP)

Gasoline Blendstock Production Diesel Blendstock Production Total Gasoline Equivalent Production Catalytic Fast Pyrolysis Oil Light Oxygenated Coproducts

Delivered Feedstock Cost Internal Rate of Return (After-Tax) On-Stream Factor

Capital Costs

100: Feedstock (Additional Dryer \& Blower Only)

200: Fast Pyrolysis \& Vapor Upgrading 300: Vapor Quench \& CFP Co-Products 400: Hydroprocessing \& Separation 500: Hydrogen Plant

600: Steam System \& Power Generation 700: Cooling Water \& Other Utilities 800: Water Management

Total Installed Equipment Cost (TIC)

Land (115 Acres at \$14000 per Acre)

Site Development

(\% of ISBL)

Indirect Costs \& Project Contingency

(\% of TIC)

Fixed Capital Investment (FCl)

Working Capital

Total Capital Investment (TCI)

$\$ 480,000$

$\$ 107,470,000$

$\$ 51,590,000$

$\$ 31,550,000$

$\$ 69,020,000$

$\$ 49,260,000$

$\$ 8,400,000$

$\$ 14,840,000$

$\$ 332,620,000$

$\$ 1,600,000$

$\$ 19,110,000$

$10.0 \%$

$\$ 220,780,000$

$66.4 \%$

$\$ 588,750,000$

$\$ 29,440,000$

$\$ 618,190,000$

Loan Interest Rate

Loan Term (Years)

$8.0 \%$

Total Installed Equipment Cost per Annual GGE

$\$ 7.47$

Fixed Capital Investment per Annual GGE

$\$ 13.23$

Plant Operating Hours per Year

7884

On-Stream Percentage

$90.0 \%$

Maximum Yield Based on HHV of Feedstock + Natural Gas Theoretical GGE Production (MM GGE / Year) 102.8

Theoretical Yield (GGE / Dry Ton)

Current Yield (Actual / Theoretical)

Overall Plant Efficiency - HHV \%

Overall Plant Efficiency - LHV \%

Version:

PyVPU-2020-V096-HT_standalone-LO-R008-copied data
\$3.03 /Gallon Gasoline Blendstock

\$3.45 /Gallon Diesel Blendstock

\$3.13 /Gallon Gasoline Equivalent (GGE)

23.0 MM Gal per Year

31.7 Gal per Dry US Ton Feedstock

20.2 MM Gal per Year

27.9 Gal per Dry US Ton Feedstock

44.5 MM GGE per Year

61.5 GGE per Dry US Ton Feedstock

$0.94 \mathrm{MM}$ bbl per Year

$1.3 \mathrm{bbl}$ per Dry US Ton Feedstock

$49.6 \mathrm{MM}$ lb per year

$3.4 \% \mathrm{w} / \mathrm{w}$ of Dry Biomass

$2.9 \%$ Acetone/Dry Biomas! $\quad 0.5 \% \mathrm{MEK} /$ Dry Biomass

$\$ 67.03$ per Dry U.S. Ton (Includes Capital Up to Throat of Pyrolyzer)

$10.0 \%$ Equity, \% of Total Investment $40.0 \%$

90.0\% CFP Oil (\$/bbl GE Product) \$131.46

\begin{tabular}{lr}
\multicolumn{2}{c}{ Operating Costs (c / GGE Product) } \\
\hline Feedstock & 108.2 \\
Natural Gas & 0.1 \\
Catalysts & 15.5 \\
Sand & 0.6 \\
Other Raw Materials & 4.0 \\
Waste Disposal & 3.3 \\
Purchased Electricity & - \\
Fixed Costs & 63.6 \\
Refinery Coprocessing Cost & - \\
Chemical Coproduct Credit & $(54.7)$ \\
Electricity Coproduct Credit & $(8.5)$ \\
Capital Depreciation & 66.2 \\
Average Income Tax & 13.9 \\
Average Return on Investment & 100.9
\end{tabular}

\begin{tabular}{|c|c|c|}
\hline \multicolumn{3}{|c|}{ Operating Costs (\$ / Year) } \\
\hline Feedstock & & $\$ 48,170,000$ \\
\hline Natural Gas & & $\$ 0$ \\
\hline Catalysts & & $\$ 6,900,000$ \\
\hline Sand & & $\$ 300,000$ \\
\hline Other Raw Mate & & $\$ 700,000$ \\
\hline Waste Disposal & & $\$ 1,460,000$ \\
\hline Purchased Elect & & $\$ 0$ \\
\hline Fixed Costs & & $\$ 28,310,000$ \\
\hline Refinery Coproc & Cost & $\$ 0$ \\
\hline Chemical Copro & redit & $-\$ 24,330,000$ \\
\hline Electricity Copro & Credit & $-\$ 3,800,000$ \\
\hline Capital Depreci & & $\$ 29,440,000$ \\
\hline Average Income & & $\$ 6,190,000$ \\
\hline Average Return & lestment & $\$ 44,900,000$ \\
\hline Total Plant Elec & Usage (kW) & 37,529 \\
\hline Electricity $\operatorname{Pr}$ & on Site (kW) & 45,771 \\
\hline Electricity $\mathrm{Pu}$ & d from Grid (kW) & 0 \\
\hline Electricity So & irid $(k W)$ & 8,242 \\
\hline Plant Electricity & (kWh /GGE) & 6.65 \\
\hline \multicolumn{3}{|c|}{ Specific Operating Conditions } \\
\hline \multirow[t]{2}{*}{ Feed Rate } & Dry Tonnes / Day & 2,000 \\
\hline & Dry Tons / Day & 2,205 \\
\hline \multirow[t]{2}{*}{ Feedstock Cost } & \$/Dry Ton & $\$ 67.03$ \\
\hline & \$/Moisture+Ash Free Ton & $\$ 68.22$ \\
\hline
\end{tabular}




\section{Table of Contents}

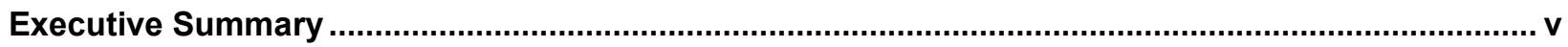

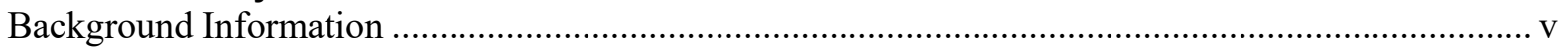

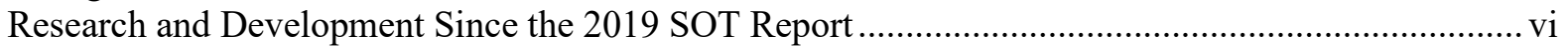

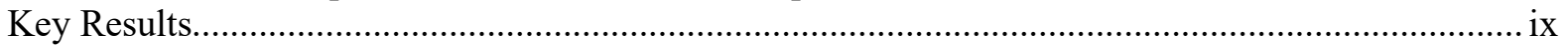

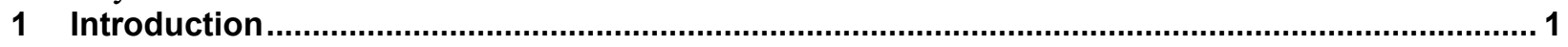

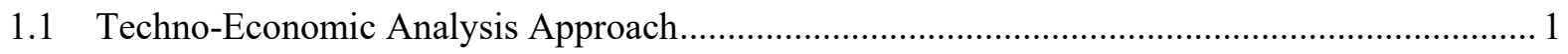

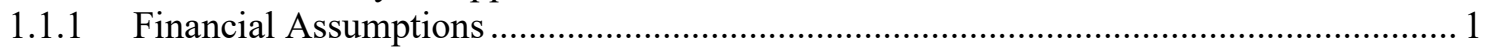

1.1.2 Estimation of Capital and Operating Costs ............................................................ 2

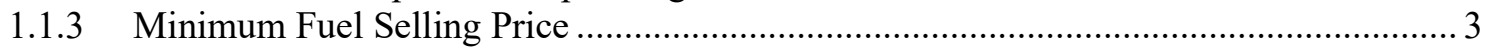

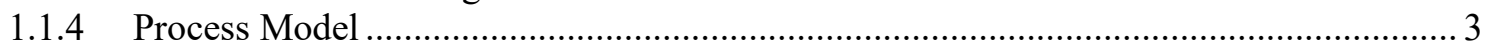

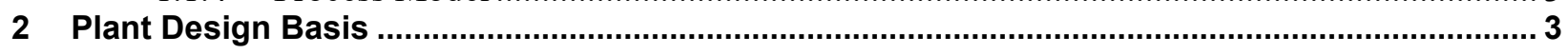

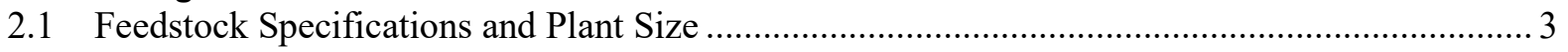

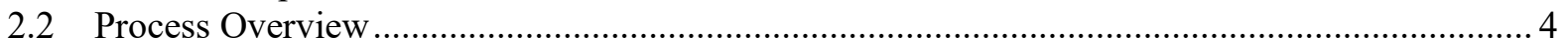

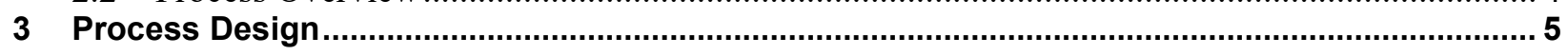

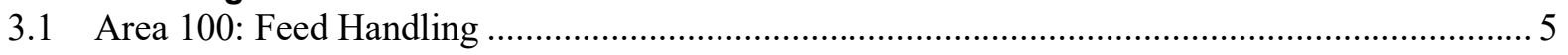

3.2 Area 200: Fast Pyrolysis and Catalytic Vapor Upgrading ................................................ 6

3.3 Area 300: CFP Product Condensation and Coproducts …....................................................... 7

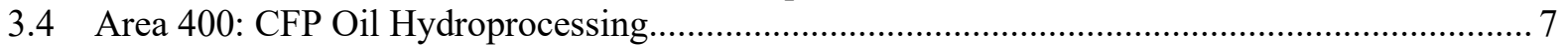

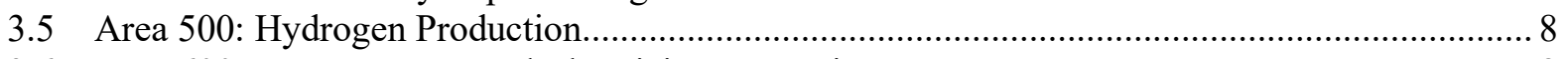

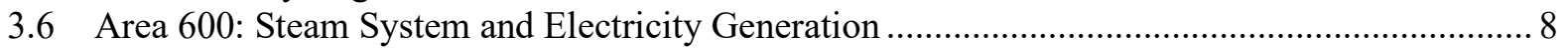

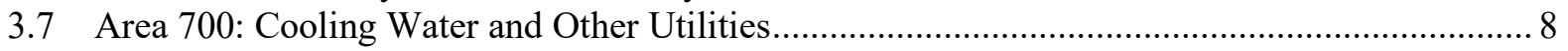

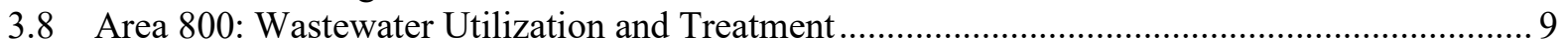

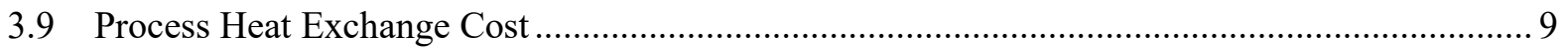

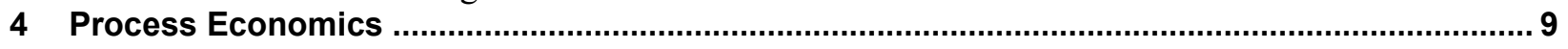

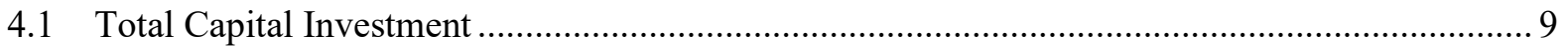

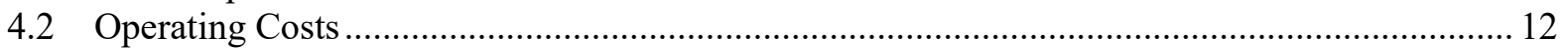

4.3 Discounted Cash Flow Analysis and the Minimum Fuel Selling Price .................................... 13

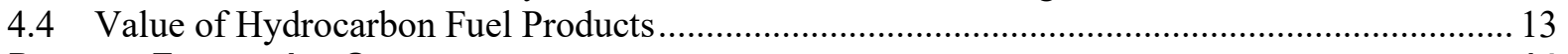

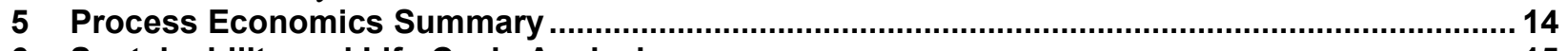

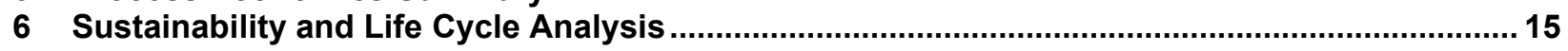

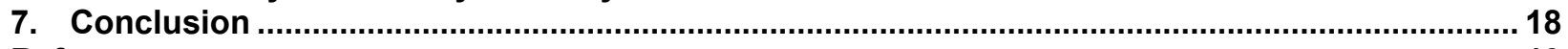

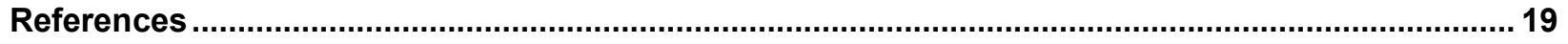

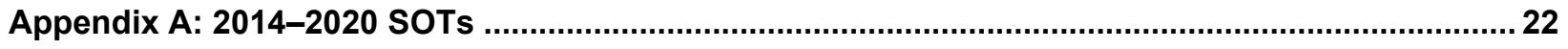




\section{List of Figures}

Figure ES-1. Modeled MFSP for 2014-2020 SOTs. There was a transition from CFP using zeolite catalysts in a fluidized bed (2014-2016) to a $\mathrm{Pt} / \mathrm{TiO}_{2}$ catalyst in a fixed bed (2017-2020).

Acetone and 2-butanone coproducts were included in 2019 and 2020. ix

Figure 1. Simplified process flow diagram for fixed-bed ex situ catalytic fast pyrolysis, coproduct

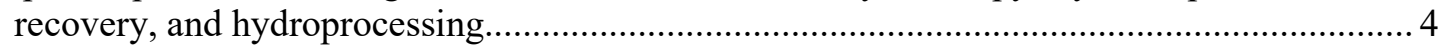

Figure 2. Cost contribution details from each process area for the 2020 target..................................... 14

Figure 3. Cost contribution details from each process area for the 2020 SOT ..................................... 14

\section{List of Tables}

Table ES-1. Key Metrics in TEA Model for the 2020 Target and SOT Cases .........................................

Table ES-2. Economic Summary (Modeled) for the 2020 SOT with Co-Hydroprocessing Option............. xi

Table ES-3. Economic Summary (Modeled) for a 2020 Sensitivity Case without 2-Butanone and Acetone

Coproducts .............................................................................................................

Table ES-4. Economic Summary (Modeled) for a 2020 Sensitivity Case with Hydroprocessing at the Biorefinery (i.e., no co-hydroprocessing) ...............................................................................ii

Table 1. Summary of Financial Assumptions for Techno-Economic Analysis ........................................ 2

Table 2. Delivered Feedstock Composition Assumptions for 50\% Forest Residues Plus 50\% Clean Pine

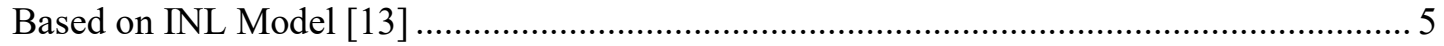

Table 3. INL-Modeled Feedstock Cost for 50\% Forest Residues Plus 50\% Clean Pine in 2016\$ [13] ....... 6

Table 4. Total Installed Equipment Costs for the 2020 SOT Case with Co-Hydroprocessing ................... 10

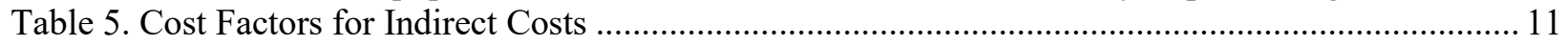

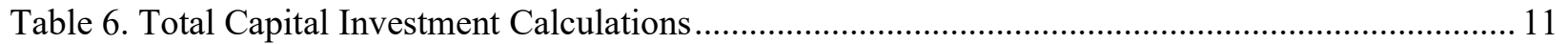

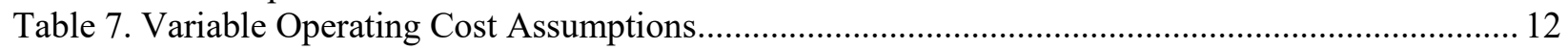

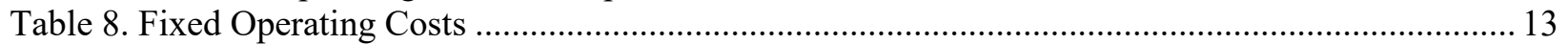

Table 9. Projected Selling Prices of Hydrocarbon Blendstocks .......................................................... 13

Table 10. Material and Energy Flows in the Conversion Process ...................................................... 15

Table 11. Sustainability and Process Efficiency Metrics for the Conversion Process, Including Co-

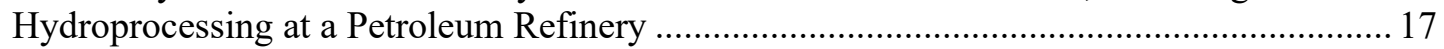

Table A-1. Processing Area Cost Contributions and Key Technical Parameters .....................................22 


\section{Introduction}

The 2015 catalytic fast pyrolysis (CFP) design report [1] detailed in situ and ex situ CFP as two potential research options for the conversion of biomass to liquid transportation fuels. Catalysts are included within the fast pyrolysis reactor in an in situ process. On the other hand, biomassderived solid material (char and inorganic matter) are separated after fast pyrolysis in an ex situ process; removal of all solids from pyrolysis vapors is desirable before catalytic upgrading in an ex situ reactor. The 2015 design report helped outline the basis for technical improvements necessary for future economic viability; associated modeled costs were presented to help understand the potential value of the research improvements [2]. Since 2015, the focus of the research has been on ex situ systems because they afford more opportunity to understand and affect the chemistry through catalyst development in the absence of biomass-derived solid material present in an in situ system. Bench-scale experimental results have been used to update the state of technology (SOT) for the ex situ pathway since 2014; significant yield improvements have been achieved via catalyst development.

Ex situ research under this project was initiated on fluidized systems with zeolite-based (primarily ZSM-5 and metal-impregnated ZSM-5) catalysts. This was based on historic precedence of experimental work documenting some of the best yields using ZSM-5 catalysts for catalytic fast pyrolysis [3]. Circulating fluidized bed systems with a combustor for coke burnoff are ideal for ZSM-5 catalysts. To broaden the research and explore other bifunctional catalyst options [4,5], such as those with noble metals in their formulations, Dutta et al. [6] proposed an analogous fixed-bed approach and analyzed its feasibility. Consequent catalyst research and associated experimental performance showed significant yield improvements using a $\mathrm{Pt} / \mathrm{TiO}_{2}$ catalyst [7]. Hence, the current process configuration for the SOT experiments since 2017 and future projections were based on the $\mathrm{Pt} / \mathrm{TiO}_{2}$ catalyst in a fixed bed as the base configuration.

The Executive Summary provides information about our research findings since the 2019 SOT update [8]; those details are not repeated here.

\subsection{Techno-Economic Analysis Approach}

The techno-economic analysis (TEA) approach for this work is similar to those detailed previously [1,6]. Overviews of process and economic assumptions and methods are provided below. Further details are available in previous process design publications $[1,6]$.

\subsubsection{Financial Assumptions}

The modeled projections in this report are based on the technology being implemented in a mature or $\mathrm{n}^{\text {th }}$ plant; additional costs associated with pioneer plants are thus not included because the purpose of this TEA is to understand the potential impact and relevance of the research in the context of future industrial implementation. A consistent set of assumptions is used for all SOTs and projections. Key assumptions are listed in Table 1. 
Table 1. Summary of Financial Assumptions for Techno-Economic Analysis

\begin{tabular}{|c|c|}
\hline Description of Assumption & Assumed Value \\
\hline Cost year & 2016 \\
\hline Internal rate of return on equity & $10 \%$ \\
\hline Plant financing by equity/debt & $40 \% / 60 \%$ of total capital investment \\
\hline Plant life & 30 years \\
\hline Income tax rate & $21 \%$ \\
\hline Interest rate for debt financing & $8.0 \%$ annually \\
\hline Term for debt financing & 10 years \\
\hline Working capital cost & $\begin{array}{r}5.0 \% \text { of fixed capital investment }(\mathrm{FCl}) \\
\text { (excluding land purchase cost) }\end{array}$ \\
\hline Depreciation schedule & 7-year MACRS a schedule [9] \\
\hline Steam plant depreciation & 20-year MACRS schedule [9] \\
\hline Construction period (spending schedule) & 3 years $(8 \%$ Y $1,60 \%$ Y $2,32 \%$ Y3) \\
\hline Plant salvage value & No value \\
\hline Startup time & 6 months \\
\hline Revenue and costs during startup & $\begin{array}{r}\text { Revenue }=50 \% \text { of normal } \\
\text { Variable costs }=75 \% \text { of normal } \\
\text { Fixed costs }=100 \% \text { of normal }\end{array}$ \\
\hline Onstream percentage after startup & $90 \%(7,884$ operating hours per year) \\
\hline
\end{tabular}

There were two significant changes to the financial assumptions compared to the previous publications [1,6]: (1) 21\% tax rate (versus a previous 35\% tax rate) and (2) 2016-dollars cost basis. These assumptions in Table 1 are consistent with the 2019 SOT report [8].

\subsubsection{Estimation of Capital and Operating Costs}

Detailed capital costs of individual equipment and their sources were listed in the 2015 design report [1], and additional fixed-bed equipment costs were presented in the subsequent analysis for fixed-bed systems $[6,8]$. Note that the fixed-bed system cost for this analysis was based on upstream reactor $\# 1$ in Dutta et al. [6], at approximately $\$ 2.5$-million base cost per $50 \%$ capacity reactor in 2013 dollars, a scaling exponent of 0.7 , and an installation factor of 1.62.

Equipment costs were scaled based on process flows in the Aspen Plus process model using a scaling exponent:

$$
\text { Scaled Equipment Cost }=\text { Base Equipment Cost }\left(\frac{\text { Scaled Capacity }}{\text { Base Capacity }}\right)^{n}
$$

The scaling exponent, $n$, is typically in the range of 0.6 to 0.7 for process equipment; however, it varies with equipment type, base size, and other factors that affect scalability. Scaling factors are documented in Appendix B of the 2015 design report [1]. 
Total installed cost (TIC) of the equipment, which includes associated piping, instrumentation and controls, electrical systems, buildings, yard improvements, and direct labor, were derived from the equipment cost by applying an installation factor $\left(f_{\text {install }}\right)$.

Total Installed Cost $($ TIC $)=f_{\text {install }} *$ Total Purchased Equipment Cost (TPEC)

Installation factors are also documented in Appendix B of the 2015 design report.

Costs were converted to 2016 dollars using:

$$
\text { Cost in } 2016 \$=\text { Base Cost }\left(\frac{2016 \text { Cost Index Value }}{\text { Base Year Cost Index Value }}\right)
$$

Operating costs were adjusted using the Producer Price Index for chemical manufacturing [10], and capital costs were adjusted using Chemical Engineering's "Plant Cost Index" [11].

The total capital investment was derived from the TIC in 2016 dollars after applying additional factors for overhead and contingency.

\subsubsection{Minimum Fuel Selling Price}

The total capital investment, along with plant operating costs, was used for a discounted cash flow analysis. Those costs, along with the gallons gasoline equivalent (GGE) of the total fuel blendstock product, were used to derive the minimum fuel selling price (MFSP) in dollars per GGE.

\subsubsection{Process Model}

The process was modeled in Aspen Plus with a detailed accounting of all mass and energy flows. Details about the Aspen Plus [12] process model for ex situ CFP were documented in the 2015 design report [1] and the subsequent fixed-bed publication [6]. The base models from the previous work were maintained for this analysis. Process assumption updates and other key aspects are described in the following sections.

\section{Plant Design Basis}

\subsection{Feedstock Specifications and Plant Size}

Feedstock information for this process was provided by Idaho National Laboratory (INL). Feedstock blend costs used for the 2020 SOT were modeled at approximately $\$ 67 /$ dry U.S. ton. The plant size was maintained at 2,000 dry metric tons per day. This is $\$ 3 /$ dry U.S. ton lower than the 2019 SOT cost, due to some reduction in modeled preprocessing costs [13].

The 2020 SOT feedstock was based on a 50/50 blend of forest residues and clean pine with a relatively high $1.75 \%$ modeled ash content (also reflected in the conversion process model by a prorated reduction of the other elements in the elemental analysis). 


\subsection{Process Overview}

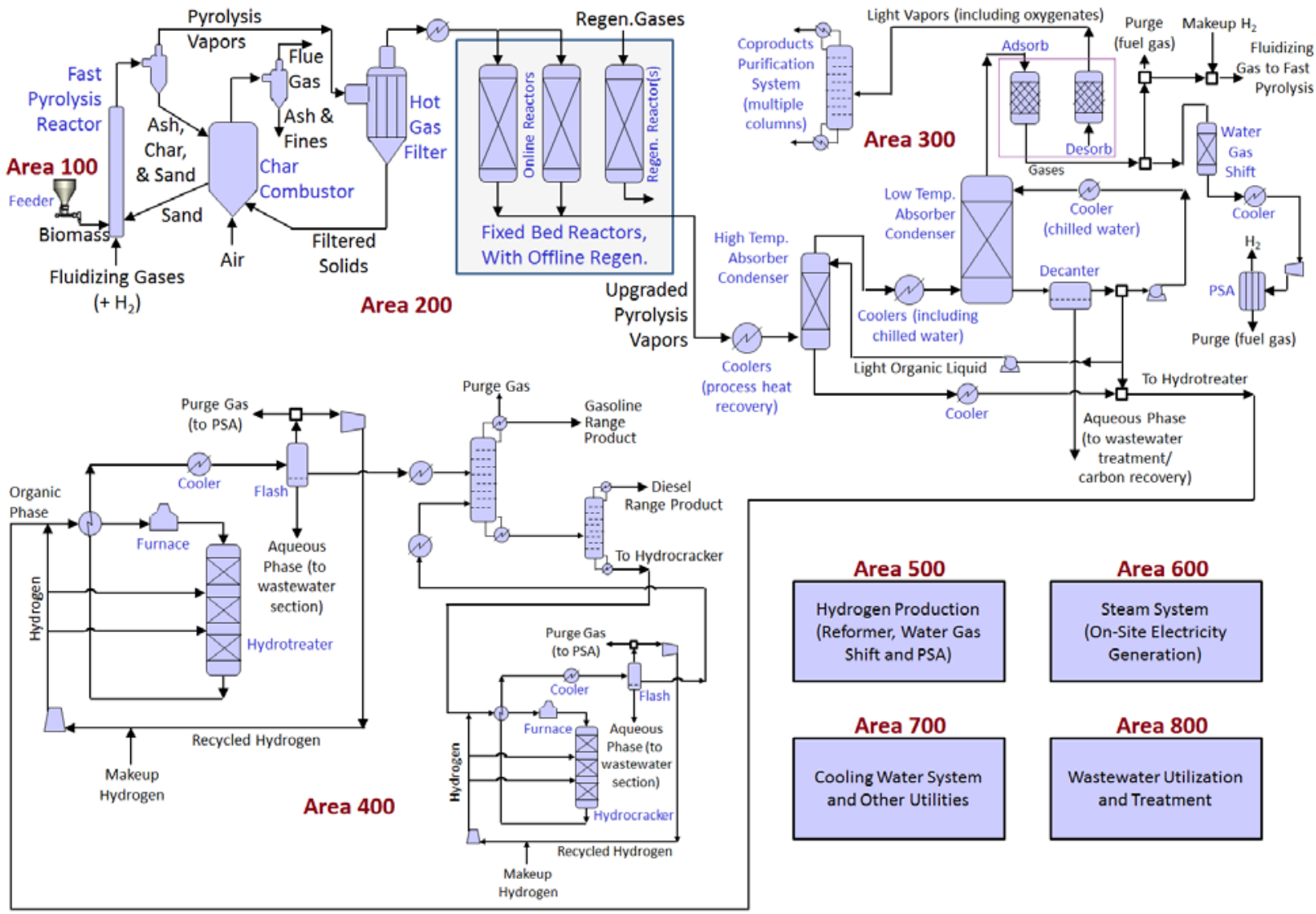

Figure 1. Simplified process flow diagram for fixed-bed ex situ catalytic fast pyrolysis, coproduct recovery, and hydroprocessing

A block flow diagram for the fixed-bed ex situ catalytic fast pyrolysis process is shown in Figure 1. The design includes eight process areas, with four core operations:

- A100: Feedstock handling (most of the processing occurs off-site and the TEA accounts for the feedstock delivered to the throat of the reactor through a cumulative cost)

- A200: Fast pyrolysis, hot gas filtration, and ex situ catalytic vapor upgrading

- A300: CFP product condensation (with the separation of the organic liquid CFP oil from an aqueous wastewater stream and use of separated permanent gases in the process). Beginning 2019, oxygenated coproducts recovery and purification was added to this area.

- A400: CFP oil hydrotreating, hydrocracking, and product distillation.

Supporting operations include:

- A500: Hydrogen production (from process off-gases)

- A600: Steam system and electricity generation (from available excess heat)

- A700: Cooling water and other utilities

- A800: Wastewater utilization and treatment (regenerative thermal oxidizer used to combust the organic content in the wastewater).

Further descriptions are included in Section 3. 


\section{Process Design}

\subsection{Area 100: Feed Handling}

The 2020 CFP conversion model requires 2,000 dry tons/day of woody feedstock. A 50\% clean pine and 50\% logging residue blend was modeled by INL, with details presented in a 2020 report [13]. All feedstock growth, handling, and processing costs are included in the cumulative feedstock costs; summary tables for composition (Table 2) and costs (Table 3) are presented below. A nominal feedstock size of $2 \mathrm{~mm}$ is specified for this process model, and necessary grinding costs are included in INL's feedstock cost. The only minor additional feedstock handling-related cost added to the conversion equipment list is a cross-flow dryer for warming the feedstock before feeding to the fast pyrolysis reactor.

The 2-inch fluidized-bed reactor (2FBR) CFP experiments at the National Renewable Energy Laboratory (NREL) also used a 50:50 blend of clean pine and residues. However, the ash content of the 50:50 blended feedstock supplied to NREL had a lower ash content compared to the $1.75 \%$ in the INL model. It is noted that we used experimental CFP yields for the TEA presented in this report; impact of ash variations is being studied separately under the FeedstockConversion Interface Consortium [14,15]. The 2020 modeled feedstock cost was slightly lower at $\$ 67.03 /$ dry ton compared to the 2019 cost of $\$ 70.15 /$ dry ton; the reduction is attributed to a small change in preprocessing costs related to additional ash removal. The impact of this feedstock cost reduction on the MFSP of the 2020 SOT base case is $\$ 0.05 /$ GGE. It is also noted that the bench-scale $2 \mathrm{FBR}$ pyrolyzer system feeds a smaller nominal particle size compared to the 2-mm size in the model, and the 2FBR pyrolyzer configuration (bubbling bed) is different from the modeled pyrolyzer configuration described in the following section.

Table 2. Delivered Feedstock Composition Assumptions for $\mathbf{5 0 \%}$ Forest Residues Plus $\mathbf{5 0} \%$ Clean Pine Based on INL Model [13]

\begin{tabular}{ll}
\hline Component & Dry wt \% \\
\hline Carbon & 50.51 \\
Hydrogen & 5.99 \\
Nitrogen & 0.17 \\
Sulfur & 0.03 \\
Oxygen & 41.55 \\
Ash & $\leq 1.75$ \\
\hline Other Metrics & \\
\hline Moisture (bulk wt \%) & 10.0 \\
Particle size (inches) & $\leq 0.08$ \\
\hline
\end{tabular}


Table 3. INL-Modeled Feedstock Cost for 50\% Forest Residues Plus 50\% Clean Pine in 2016\$ [13]

\begin{tabular}{lr}
\hline \multicolumn{2}{c}{ Cost Summary (\$/Dry U.S. Ton) (2016\$) } \\
\hline 2022 Projection \\
\hline Grower payment & $\$ 9.74$ \\
Harvest and collection & $\$ 4.94$ \\
Field-side preprocessing & $\$ 8.41$ \\
Transportation & $\$ 12.22$ \\
Preprocessing & $\$ 25.43$ \\
Storage & $\$ 0.68$ \\
Handling & $\$ 2.65$ \\
Preprocessing construction & $\$ 2.96$ \\
\hline Grand Total & $\$ 67.03$ \\
\hline
\end{tabular}

\subsection{Area 200: Fast Pyrolysis and Catalytic Vapor Upgrading}

The process model for Area 200 includes a circulating fluidized bed fast pyrolysis reactor. The dual-bed reactor system includes a riser reactor for fast pyrolysis of biomass at approximately $500^{\circ} \mathrm{C}\left(932^{\circ} \mathrm{F}\right)$, with short biomass residence times of approximately 2 seconds in the riser and a char combustor for providing heat to the endothermic fast pyrolysis reactions. Circulating sand is heated in the char combustor and sent to the riser reactor, where it heats the biomass to pyrolysis temperatures. The solids (char and mineral matter) from fast pyrolysis are removed from the hot vapors by cyclones. An additional hot gas filter is also included to remove any residual solids. This hot gas filter is necessary because of the downstream fixed-bed ex situ catalytic vapor upgrading reactor that can easily plug from any residual solids. The catalytic fixed-bed reactor system includes a $\mathrm{Pt} / \mathrm{TiO}_{2}$ catalyst with $0.5 \mathrm{wt} \% \mathrm{Pt}$ loading. A 2-year catalyst lifetime is assumed in the model, along with a $70 \%$ cost recovery at the end of 2 years. A catalyst cost model called CatCost [16], developed under the Chemical Catalysis for Bioenergy Consortium [17], was used to estimate the cost of the $\mathrm{Pt} / \mathrm{TiO}_{2}$ catalyst. Note that the 2015 design report [1] included a circulating fluidized bed ex situ reactor design with zeolite catalyst.

The CFP bench-scale experimental setup (2FBR system) and analytical methods used to generate experimental results are described by Griffin et al. [7]. As a brief overview, a 2-inch fluidized pyrolyzer was followed by a hot gas filter and a fixed-bed $\mathrm{Pt} / \mathrm{TiO}_{2}$ catalyst vapor upgrading reactor. It was noted earlier that the $2 \mathrm{FBR}$ system was rebuilt and recommissioned prior to the 2020 SOT experiments.

The blend of 50\% forest residues and $50 \%$ clean pine was converted at a fast pyrolysis temperature of $500^{\circ} \mathrm{C}$, the fixed-bed ex situ reactor set point temperature was maintained at $400^{\circ} \mathrm{C}$, and the biomass-to-catalyst ratio was 12 . The catalyst had $0.5 \mathrm{wt} \% \mathrm{Pt}$ on a $\mathrm{TiO}_{2} \mathrm{support}$ CFP oil with an oxygen content of $16.6 \mathrm{wt} \%$ on dry basis was produced with a carbon yield of $35.7 \%$. The coke formation on the catalyst was $1.7 \mathrm{wt} \%$. All these results are similar (but not identical) to the fiscal year (FY) 2019 SOT and show that results could be replicated using the updated 2FBR system. The largest variation was seen in the amount of biomass carbon lost to the aqueous phase, which dropped from $4.4 \%$ in FY 2019 to $1.7 \%$ (modeled at 1.8\%) in FY 2020, 
very likely attributed to some changes made to the experimental condensation train. This helped lower the MFSP because of reduced wastewater treatment costs.

In addition to the CFP oil, nearly $13 \%$ of the biomass carbon was present in light condensables. There was high selectivity to three specific compounds, with carbon yields of approximately $4.3 \%$ acetone, $3.5 \%$ acetaldehyde, and $1.4 \%$ 2-butanone. The combined carbon yield for the CFP oil and light condensables was nearly 49\%. Additional metrics are shown in Table ES-1.

\subsection{Area 300: CFP Product Condensation and Coproducts}

The process design for this section was maintained from the 2015 design report [1]. The system consists of two direct quench absorber/condensers. The upgraded vapors from the ex situ reactors are initially cooled via indirect heat exchange up to the modeled dew point of the vapor stream. A heavy organic liquid is then condensed in the first absorber/condenser; the light organic liquid product from the second condenser is used as the quench liquid. The uncondensed light vapors from the first condenser, as well as the vaporized quench liquid, are then sent through heat exchangers. The partially condensed vapors enter the second absorber/condenser column for a final quench using a stream of recycled (and cooled) light organic liquid. The bottom product of the second condenser is separated into an aqueous waste stream (sent to Area 800) and an organic product. As previously mentioned, part of this light organic liquid product is also recycled for use as a quench liquid for both the absorber/condensers. In this design, the heavy organic liquid from the first condenser and light organic liquid from the second condenser are mixed and sent to Area 400 for hydroprocessing or to a petroleum refinery for cohydroprocessing.

As mentioned in the Executive Summary, major additional modeling was included in the FY 2020 SOT to help understand the recovery, purity, and energy consumption to get saleable acetone and 2-butanone coproducts. In the process design, the stream is sent to an adsorption system to remove the light oxygenates and other species, while letting the lighter gases pass through. The adsorbed species are then desorbed in a swing system. The desorbed stream is sent to a series of distillation columns and liquid-liquid extraction systems to purify acetone and 2butanone from other adsorbed species. The adsorption/desorption system cost was estimated from an ethanol dehydration mol-sieve system documented by Humbird et al. [18]. The scaling variable was moles of adsorbed species adjusted for partial pressure. Additional contingencies were added to cover potential cost escalations from our current estimates. There is also a difference in the desorption carrier medium between the experiments and the process model, and additional experiments will be conducted to experimentally test the same medium specified in the model. The purification of the mixed desorbed stream to obtain chemical-grade acetone and 2-butanone was modeled in Aspen Plus (as discussed in the Executive Summary).

\subsection{Area 400: CFP Oil Hydroprocessing}

The 2015 design report was written based on a premise that a single reactor system can handle the hydrotreating of the CFP organic liquid. Experiments in 2017-2019 proved that this is a valid assumption, and it is possible to get to less than $1 \%$ oxygen content in the CFP oil after a single hydrotreating step. This FY 2020 target and SOT cases extended the process design to include co-hydrotreating of CFP oil with petroleum streams. Specifically, experiments were conducted by co-feeding CFP oil with straight-run diesel at a 10:90 vol \% ratio for this 2020 SOT. A 
discussion of key achievements related to co-hydrotreating is included in the Executive Summary. The remainder of this section focuses on our methodology for assessing cohydroprocessing costs.

The stand-alone hydroprocessing section of the Aspen Plus model was designed to predict hydrogen makeup consumption for any given CFP oil oxygen content and associated hydroprocessing carbon efficiency. The hydrogen consumption attributed to the CFP oil during co-hydroprocessing can thus be derived using the same model using the experimental interpretation of carbon efficiency for the CFP oil portion and the measured oxygen content of the CFP oil. The makeup hydrogen for hydroprocessing in petroleum refineries was correlated with various associated operating expenses from published petroleum refining literature. Those correlations, along with the modeled hydrogen consumption, provided estimates for prorating expenses attributed to hydroprocessing the CFP oil portion of the feed. We used this approach because of the disproportionate presence of heteroatoms (oxygen) in the CFP oil relative to most petroleum streams, and hydrogen use is a good indicator of heteroatom removal and associated expenses. For the FY 2020 SOT base case, the co-hydroprocessing expense was $\$ 0.226 / \mathrm{GGE}$. We anticipate publishing more details of our estimation methods separately in the future. Capital costs for co-hydroprocessing were not included in the estimates because of the assumption of using existing equipment at the petroleum refinery.

\subsection{Area 500: Hydrogen Production}

Hydrogen demands at the biorefinery were met (in the process models) without importing additional natural gas. Off-gases, primarily from CFP and other parts of the process, were processed in a steam reformer to produce hydrogen, and purified hydrogen was produced using pressure swing adsorption units. Process design details for Area 500 are consistent with the 2015 design report [1]. For co-hydroprocessing of the CFP oil at a petroleum refinery, we assumed steam reforming of natural gas for hydrogen production. This use of natural gas at the petroleum refinery has a significant greenhouse gas $(\mathrm{GHG})$ footprint (as mentioned in other sections of this report).

\subsection{Area 600: Steam System and Electricity Generation}

Heat available in the modeled process was used to generate electricity. Excess electricity, after meeting process demands, was sold to the grid. Process design details are consistent with the 2015 design report [1]. For off-site co-hydroprocessing at a petroleum refinery (as in this 2020 SOT case), off-gases used for hydrogen production in the hydrogen self-sufficient stand-alone hydroprocessing case become available for additional electricity production.

\subsection{Area 700: Cooling Water and Other Utilities}

Air cooling was the major cooling method in the process design when in-process heat recovery was not feasible. Process heat exchange and air cooling costs are included with the costs of the respective process areas. Water cooling was used primarily for cooling process streams below $140^{\circ} \mathrm{F}$; process stream temperatures of $110^{\circ} \mathrm{F}$ were achieved after water cooling. Chilled water was used for cooling below $110^{\circ} \mathrm{F}$. Process design details for Area 700 are consistent with the 2015 design report [1]. 


\subsection{Area 800: Wastewater Utilization and Treatment}

Organic species in the wastewater stream were oxidized in a regenerative thermal oxidizer to allow discharge of the stream contents in an environmentally acceptable manner.

\subsection{Process Heat Exchange Cost}

A detailed heat exchange network was developed for the ex situ process and documented in the 2015 design report [1]. Cost estimates for the heat exchange networks in subsequent models were derived by scaling the costs from the design report using the total process heat exchange duty as the scaling basis. Pinch analysis was done to ensure that the process model is thermodynamically valid; the minimum approach temperature for the 2020 SOT base case was $14.5^{\circ} \mathrm{F}$.

\section{Process Economics}

Capital and operating costs are listed in this section. Note that most of the information presented here is based on previously documented details [1,6]. Costs were updated to a 2016-dollars basis and scaled based on specific stream flows in the process models (as stated in Section 1.1.2). Details from the 2020 target and SOT models are presented below. Information for the other models can be obtained from the tables in the Executive Summary and the Appendix.

\subsection{Total Capital Investment}

Installed capital costs are shown in Table 4, with per-area total purchased equipment cost (TPEC), installation factors, and TIC. 
Table 4. Total Installed Equipment Costs for the 2020 SOT Case with Co-Hydroprocessing

\begin{tabular}{|c|c|c|c|c|c|c|c|}
\hline \multirow[b]{2}{*}{ Area } & \multirow[b]{2}{*}{ Process Description } & \multicolumn{3}{|c|}{2020 Target } & \multicolumn{3}{|c|}{2020 SOT } \\
\hline & & $\begin{array}{l}\text { TPEC } \\
\text { (MM\$) }\end{array}$ & $\mathbf{f}_{\text {install }}$ & $\begin{array}{r}\text { TIC } \\
\text { (MM\$) }\end{array}$ & $\begin{array}{l}\text { TPEC } \\
\text { (MM\$) }\end{array}$ & $\mathbf{f}_{\text {install }}$ & $\begin{array}{r}\text { TIC } \\
\text { (MM\$) }\end{array}$ \\
\hline 100 & Feed handling and drying a & 0.3 & 1.96 & 0.6 & 0.3 & 1.96 & 0.5 \\
\hline 200 & $\begin{array}{l}\text { Fast pyrolysis and vapor } \\
\text { upgrading }\end{array}$ & 44.7 & 2.52 & 112.4 & 45.2 & 2.37 & 107.3 \\
\hline 300 & $\begin{array}{l}\text { Pyrolysis vapor quench, } \\
\text { condensation, and coproduct } \\
\text { recovery }\end{array}$ & 24.9 & 1.84 & 45.8 & 28.6 & 1.80 & 51.3 \\
\hline 400 & $\begin{array}{l}\text { Hydroprocessing and product } \\
\text { separation }\end{array}$ & $0^{b}$ & - & $0^{b}$ & $0^{b}$ & - & $0 \mathrm{~b}$ \\
\hline 500 & Hydrogen plant & 24.8 & 2.03 & 50.1 & 25.0 & 2.04 & 51.1 \\
\hline 600 & $\begin{array}{l}\text { Steam system and power } \\
\text { generation }\end{array}$ & 33.0 & 1.84 & 60.8 & 33.2 & 1.84 & 61.1 \\
\hline 700 & Cooling water and other utilities & 4.4 & 2.02 & 8.9 & 4.2 & 2.02 & 8.6 \\
\hline 800 & $\begin{array}{l}\text { Wastewater management and } \\
\text { recycle }\end{array}$ & 10.1 & 2.34 & 23.6 & 6.2 & 2.35 & 14.5 \\
\hline \multicolumn{2}{|c|}{ ISBL c (Areas 100-400) } & 69.9 & 2.27 & 158.8 & 74.0 & 2.15 & 159.1 \\
\hline \multicolumn{2}{|c|}{ OSBL d (Areas 500-800) } & 72.3 & 1.98 & 143.5 & 68.5 & 1.97 & 135.2 \\
\hline \multicolumn{2}{|l|}{ Total } & 142.2 & 2.13 & 302.3 & 142.6 & 2.06 & 294.3 \\
\hline
\end{tabular}

a Most investment costs for feed handling and drying are included in the per-unit woody feedstock price. This cost is for a secondary biomass dryer that serves to recover heat.

b Capital at the biorefinery is zero because co-hydroprocessing occurs at the petroleum refinery in this model, and we assume the reuse of existing equipment.

${ }^{c}$ Inside battery limits

d Outside battery limits.

The estimated TPEC, $f_{\text {install, }}$ and TIC for the light oxygenates recovery and purification section in the base model (included above under Area 300 ) were $\$ 17$ million, 1.82, and $\$ 30.8$ million, respectively. As mentioned earlier, the reduced carbon loss to the aqueous phase in the 2020 SOT resulted in capital cost savings reflected by reduced Area 800 costs.

The sum of equipment purchases and installation/construction costs is defined as the total direct cost. Indirect costs such as project management and engineering, procurement, and construction services are estimated with factors on the total direct cost, as shown in Table 5. 
Table 5. Cost Factors for Indirect Costs

\begin{tabular}{lr}
\hline Indirect Costs & \% of Total Direct Cost \\
\hline Prorated expenses & 10.0 \\
Home office and construction fees & 20.0 \\
Field expenses & 10.0 \\
Project contingency & 10.0 \\
Other costs (startup and permits) & 10.0 \\
\hline Total Indirect Costs & $\mathbf{6 0 . 0}$ \\
\hline a Excluding land purchase cost.
\end{tabular}

The sum of direct and indirect costs is defined as the fixed capital investment (FCI). The working capital is estimated to be $5 \%$ of the FCI. The sum of FCI and working capital is the total capital investment. Table 6 presents a summary of these capital quantities for the 2020 target and SOT cases.

Table 6. Total Capital Investment Calculations

\begin{tabular}{|c|c|c|c|}
\hline & & 2020 Target & 2020 SOT \\
\hline TPEC & & $\$ 142,210,000$ & $\$ 142,560,000$ \\
\hline Installation factor & & 2.126 & 2.064 \\
\hline TIC & & $\$ 302,330,000$ & $\$ 294,270,000$ \\
\hline \multicolumn{4}{|l|}{ Other direct costs } \\
\hline Land (not depreciated) & & $\$ 1,610,000$ & $\$ 1,610,000$ \\
\hline Warehouse & $4.0 \%$ of ISBL & $\$ 6,350,000$ & $\$ 6,360,000$ \\
\hline Site development & $10.0 \%$ of ISBL & $\$ 15,880,000$ & $\$ 15,910,000$ \\
\hline Additional piping & $4.5 \%$ of ISBL & $\$ 7,150,000$ & $\$ 7,160,000$ \\
\hline Total direct costs & & $\$ 331,710,000$ & $\$ 323,700,000$ \\
\hline Indirect costs & $\begin{array}{r}\% \text { of total direct cost } \\
\text { (excluding land) }\end{array}$ & & \\
\hline Prorated expenses & $10.0 \%$ & $\$ 33,170,000$ & $\$ 32,370,000$ \\
\hline Home office and construction fees & $20.0 \%$ & $\$ 66,340,000$ & $\$ 64,740,000$ \\
\hline Field expenses & $10.0 \%$ & $\$ 33,170,000$ & $\$ 32,370,000$ \\
\hline Project contingency & $10.0 \%$ & $\$ 33,170,000$ & $\$ 32,370,000$ \\
\hline Other costs (startup and permits) & $10.0 \%$ & $\$ 33,170,000$ & $\$ 32,370,000$ \\
\hline Total indirect costs & $60.0 \%$ & $\$ 199,030,000$ & $\$ 194,220,000$ \\
\hline $\mathrm{FCl}$ & & $\$ 530,740,000$ & $\$ 517,920,000$ \\
\hline Working capital & $\begin{array}{r}5.0 \% \text { of } \mathrm{FCl} \\
\text { (excluding land) }\end{array}$ & $\$ 26,540,000$ & $\$ 25,900,000$ \\
\hline Total capital investment (TCI) & & $\$ 557,280,000$ & $\$ 543,820,000$ \\
\hline TCI/TPEC & & 3.919 & 3.815 \\
\hline FCI Lang Factor $=\mathrm{FCl} / \mathrm{ISBL}$ TPEC & & 7.592 & 6.997 \\
\hline $\mathrm{TCI}$ Lang Factor $=\mathrm{TCI} / \mathrm{ISBL}$ TPEC & & 7.972 & 7.347 \\
\hline
\end{tabular}




\subsection{Operating Costs}

Variable operating cost assumptions are shown in Table 7. The major addition to the table in the 2015 design report [1] is the $\mathrm{Pt} / \mathrm{TiO}_{2}$ catalyst used in the fixed-bed ex situ reactor.

Consumption/discharge quantities are shown in Table 10, and the economic summaries for the different cases in the Executive Summary provide further details.

Table 7. Variable Operating Cost Assumptions

\begin{tabular}{|c|c|}
\hline Variable & Information and Operating Cost (cost year in parentheses) \\
\hline \multirow{2}{*}{$\begin{array}{l}\text { Fluidized bed } \\
\text { media }\end{array}$} & For fast pyrolysis, the bed medium is sand. \\
\hline & Sand price: $\$ 45.74 /$ U.S. ton $(2011 \$)$ [19]; initial fill, then make up for attrition. \\
\hline $\begin{array}{l}\text { Fixed-bed ex situ } \\
\text { vapor upgrading } \\
\text { catalyst }\end{array}$ & $\begin{array}{l}\mathrm{Pt} / \mathrm{TiO}_{2} \text { with } 0.5 \% \mathrm{Pt} \text { loading. Catalyst unit cost } \$ 92.35 / \mathrm{lb}(2014 \$) \text { [16]. A 2-year } \\
\text { lifetime is assumed, with } 70 \% \text { cost recovery at the end of } 2 \text { years. Quantity of } \\
\text { catalyst was determined using a weight hourly space velocity of } 5 \mathrm{~h}^{-1} \text { based on } \\
\text { vapor flow and an additional } 70 \% \text { overdesign. }\end{array}$ \\
\hline \multirow[t]{2}{*}{$\begin{array}{l}\text { Hydrotreating and } \\
\text { hydrocracking } \\
\text { catalysts }\end{array}$} & $\begin{array}{l}\text { To determine the amount of catalyst inventory, the hydroprocessors were sized for } \\
\text { a weight hourly space velocity of } 0.5 \mathrm{~h}^{-1} \text { based on the expected hydroprocessing } \\
\text { severity. Initial fill is then replaced every } 2 \text { years. }\end{array}$ \\
\hline & $\begin{array}{l}\text { Price: } \$ 20 / \mathrm{lb}(2011 \$) \text { based on NREL calculations using metals pricing and costs } \\
\text { for manufacturing processes and some buffer for modifications. }\end{array}$ \\
\hline \multirow{2}{*}{$\begin{array}{l}\text { Steam methane } \\
\text { reformer catalysts }\end{array}$} & Based on a literature value of price per unit hydrogen produced. \\
\hline & Price: $\$ 7.80 /$ U.S. ton hydrogen $(2011 \$)[20]$ \\
\hline \multirow[t]{3}{*}{ Natural gas } & $\begin{array}{l}\text { Purchased from pipeline for feed to steam methane reformer for hydrogen } \\
\text { production. Natural gas has an insignificant cost contribution because of minimal } \\
\text { process use. }\end{array}$ \\
\hline & $\begin{array}{l}\text { Price at biorefinery: } \$ 239 / \text { U.S. ton ( } \$ 5 \text { per million British thermal units [MMBtu]) } \\
(2011 \$) \text {. }\end{array}$ \\
\hline & $\begin{array}{l}\text { For the co-hydroprocessing case, natural gas was } \$ 3.5 / M M B t u \text {, based on a } 10- \\
\text { year average for Texas using information from the U.S. Energy Information } \\
\text { Administration (https://www.eia.gov/naturalgas/). }\end{array}$ \\
\hline Solids disposal & Price: $\$ 33 /$ tonne (1998\$) [21]. \\
\hline \multirow[t]{2}{*}{ Diesel fuel } & Usage: $10 \mathrm{gal} / \mathrm{h}$ plantwide use. \\
\hline & $\begin{array}{l}2012 \text { price projection: } \$ 21.29 / \mathrm{MMBtu}(2009 \$)[22]=\$ 2.86 / \text { gal at } 0.85 \text { specific } \\
\text { gravity. }\end{array}$ \\
\hline Water makeup & Price: $\$ 0.22 /$ tonne $(2001 \$)[23]=\$ 0.20 /$ U.S. ton. \\
\hline \multirow[t]{4}{*}{ Chemicals } & Boiler feedwater chemicals_-Price: $\$ 75 / \mathrm{MM} \mathrm{lb}$ blowdown (2014 vendor estimate). \\
\hline & $\begin{array}{l}\text { Cooling tower chemicals-Price: } \$ 33.84 / y r \text { per U.S. ton of cooling capacity (2014 } \\
\text { vendor estimate). }\end{array}$ \\
\hline & Caustic_Price: $\$ 150 /$ dry U.S. ton (2010\$) [18]. \\
\hline & $\begin{array}{l}\text { Prices of solvents for light oxygenates purification-Solvent } 1: \$ 1.2 / \mathrm{lb} \text {; Solvent } 2 \text { : } \\
\$ 0.4 / \mathrm{lb} \text {. }\end{array}$ \\
\hline \multirow[t]{2}{*}{ Wastewater } & $\begin{array}{l}\text { Most wastewater is cleaned using a reverse osmosis system and recycled. } \\
\text { Additional treatment is assumed for the balance. }\end{array}$ \\
\hline & Price: \$0.022/gallon (2011\$). Based on Humbird et al. [18]. \\
\hline
\end{tabular}

Note: Costs shown were updated to 2016 dollars using the Producer Price Index for chemical manufacturing [10]. 
Fixed operating costs related to salaries are shown in Table 8. Number of personnel and their salaries were maintained from the 2015 design report [1], and salaries were adjusted using a labor cost index [24].

Table 8. Fixed Operating Costs

\begin{tabular}{lrrr}
\hline Cost Item & Factor & 2020 Target & 2020 SOT \\
\hline Salaries & & $\$ 3,440,000$ & $\$ 3,440,000$ \\
Benefits and overhead & $90.0 \%$ of total salaries (after adding $10 \%)$ & $\$ 3,100,000$ & $\$ 3,100,000$ \\
Maintenance & $3.0 \%$ of fixed capital investment $(\mathrm{FCl}$ a) & $\$ 15,922,000$ & $\$ 15,538,000$ \\
Insurance and taxes & $0.7 \%$ of fixed capital investment $\left(\mathrm{FCl}{ }^{a}\right)$ & $\$ 3,715,000$ & $\$ 3,625,000$ \\
\hline Total fixed operating costs (2016\$/year) & $\mathbf{\$ 2 6 , 2 0 0 , 0 0 0}$ & $\mathbf{\$ 2 5 , 7 0 0 , 0 0 0}$ \\
\hline
\end{tabular}

a Percentages of $\mathrm{FCl}$ exclude land purchase cost.

\subsection{Discounted Cash Flow Analysis and the Minimum Fuel Selling Price}

Once the capital and operating costs are determined, the GGE of fuel production is used to calculate an MFSP (in \$/GGE) using a discounted cash flow rate of return (DCFROR) analysis. Further details are available in the 2015 design report [1].

\subsection{Value of Hydrocarbon Fuel Products}

The results of the cash flow analyses for the 2020 target and SOT cases are summarized in Table 9. Gasoline and diesel are normalized by lower heating value (LHV) to represent a single gasoline-equivalent product and MFSP. To calculate individual selling prices for gasoline and diesel, the MFSP per GGE is ratioed back to these products by lower heating value. The densities of the blendstocks were assumed to be those of U.S. conventional gasoline $(2,819 \mathrm{~g} / \mathrm{gal}$ or 6.215 $\mathrm{lb} / \mathrm{gal})$ and diesel fuels $(3,167 \mathrm{~g} / \mathrm{gal}$ or $6.982 \mathrm{lb} / \mathrm{gal})$ [25].

Table 9. Projected Selling Prices of Hydrocarbon Blendstocks

\begin{tabular}{lrr}
\hline & 2020 Target & 2020 SOT \\
\hline $\begin{array}{l}\text { Lower heating value for gasoline-range products } \\
\text { (simulation result) }\end{array}$ & $111,862 \mathrm{Btu} / \mathrm{gal}$ & $112,335 \mathrm{Btu} / \mathrm{gal}$ \\
$\begin{array}{l}\text { Lower heating value for diesel-range products } \\
\text { (simulation result) }\end{array}$ & $126,739 \mathrm{Btu} / \mathrm{gal}$ & $127,855 \mathrm{Btu} / \mathrm{gal}$ \\
Calculated gasoline-equivalent MFSP & $\mathbf{\$ 3 . 0 9 / G G E}$ & $\mathbf{\$ 2 . 8 3 / G G E}$ \\
Calculated actual MFSP for gasoline-range products & $\mathbf{\$ 2 . 9 8 / g a l}$ & $\mathbf{\$ 2 . 7 4 / g a l}$ \\
Calculated actual MFSP for diesel-range products & $\mathbf{\$ 3 . 3 7 / g a l ~}$ & $\mathbf{\$ 3 . 1 2 / g a l}$ \\
Gasoline lower heating value for GGE normalization & & $116,090 \mathrm{Btu} / \mathrm{gal}$ \\
\hline
\end{tabular}




\section{Process Economics Summary}

The contribution of individual areas toward the MFSP on a dollar-per-GGE basis are shown in Figure 2 (2020 target) and Figure 3 (2020 SOT).

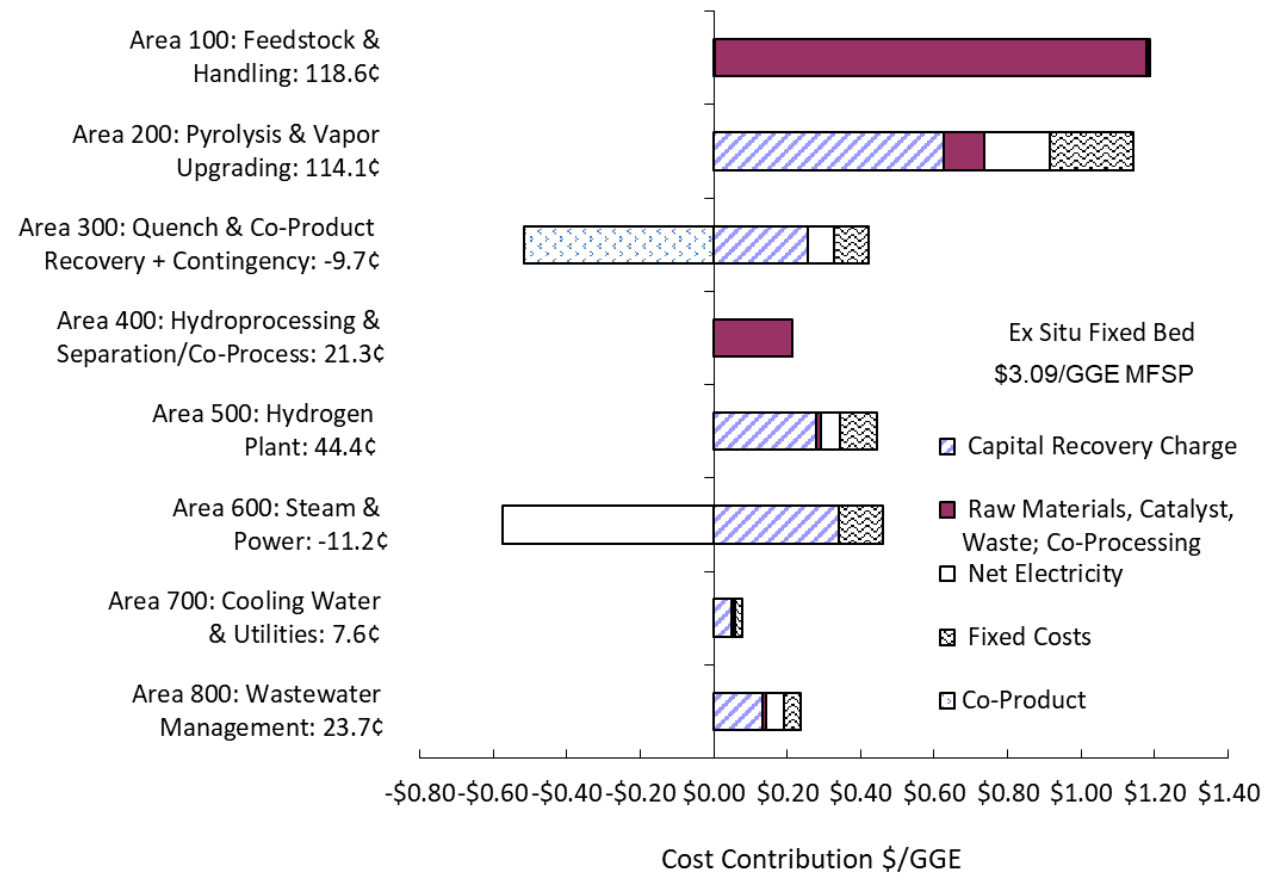

Figure 2. Cost contribution details from each process area for the 2020 target

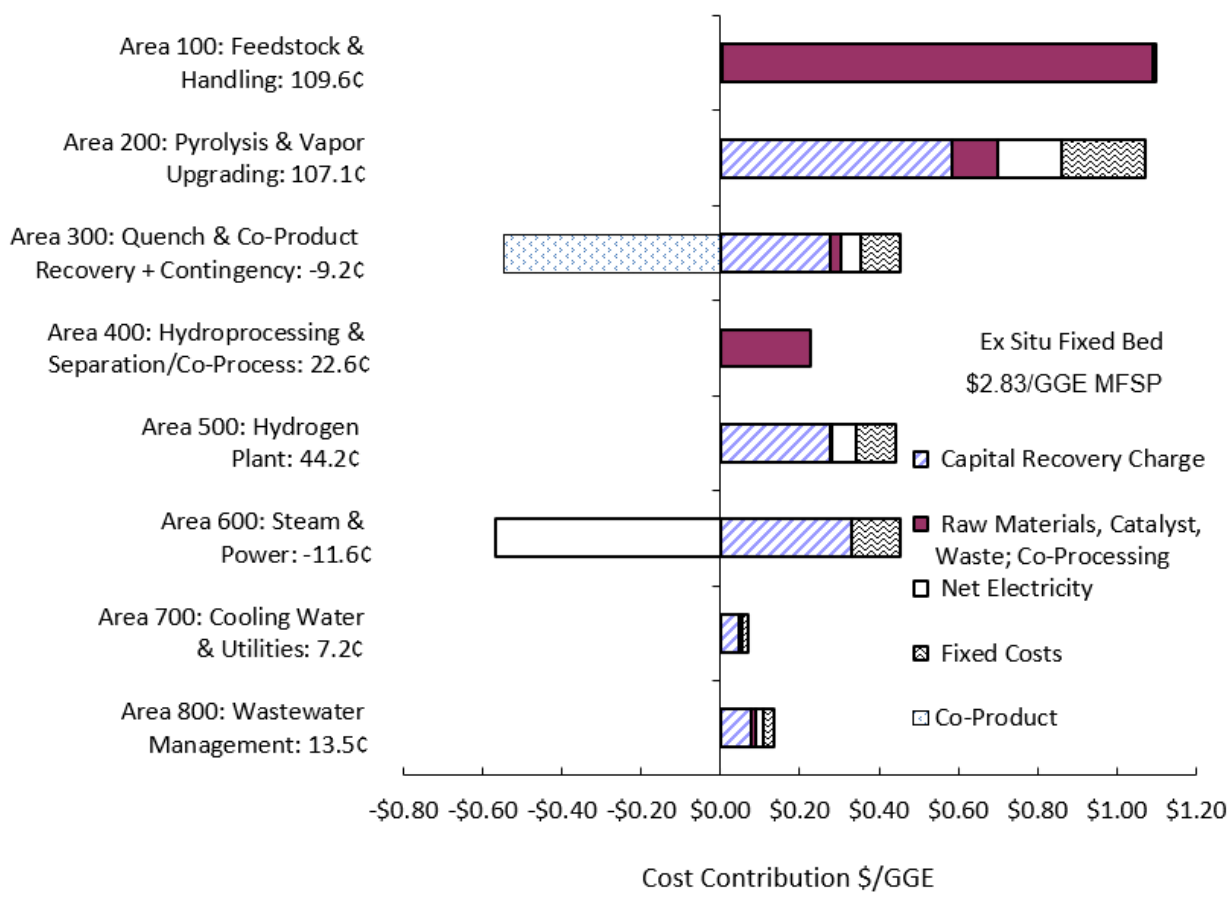

Figure 3. Cost contribution details from each process area for the 2020 SOT 
Note the biggest changes in the 2020 SOT case presented above compared to the previous 2020 target/projection: (1) savings from higher yield, thus dividing all expenses by a larger GGE value; (2) additional electricity generation at the biorefinery because we now know that heat exchange during coke regeneration can help produce high-quality steam (this heat was previously not counted as usable); (3) removal of very conservative contingencies for light oxygenates separation, which were included in the target because the 2020 target model was not fully developed for light oxygenates purification; (4) a lower feedstock cost of $\$ 67 /$ dry U.S. ton (vs. \$70/dry U.S. ton); and (5) lower wastewater treatment costs.

\section{Sustainability and Life Cycle Analysis}

This section presents sustainability metric indicators for the 2020 SOT conversion process models. Direct air emissions from the biorefinery $\left(\mathrm{CO}_{2}, \mathrm{NO}_{2}\right.$, and $\left.\mathrm{SO}_{2}\right)$, water consumption, and other process-related metrics were taken from the Aspen Plus models. The material and energy flow information from the conversion models also help capture the impacts of input raw materials, and outputs such as fuel yields, waste, and coproducts. Pertinent flows are shown in Table 10 .

The input/output inventories in Table 10 also provide the necessary information required for performing life cycle and supply chain sustainability modeling to quantify GHG emissions and fossil energy consumption. The biorefinery GHGs and fossil energy consumption are quantified separately under supply chain sustainability analysis (SCSA) efforts by Argonne National Laboratory (ANL) [26]. A complete supply chain life cycle analysis is required to understand the sustainability implications of this technology pathway and quantify associated reduction in GHG emissions from the production of the biomass-derived liquid fuel blendstock (compared to petroleum-derived liquid fuels).

Table 10. Material and Energy Flows in the Conversion Process

\begin{tabular}{lrr}
\hline & 2020 Target & 2020 SOT \\
\hline Products & & \\
Gasoline fuel (lb/h) & 18,189 & 18,123 \\
Diesel fuel (Ib/h) & 16,913 & 17,896 \\
Byproducts & & \\
Excess electricity (kW) & 20,031 & 36,019 \\
Methyl-ethyl-ketone (MEK) (Ib/h) & 1178 & 924 \\
Acetone (Ib/h) & 5,046 & 5,366 \\
\hline Resource Consumption at Petroleum Refinery & Flow rate & Flow rate \\
& & \\
\hline Fuel natural gas (MMBtu/h) & 239 & 262 \\
Electricity (kW) & 2,719 & 2,986 \\
Steam (Ib/h) & $-4,507$ & $-4,697$ \\
Cooling water makeup (gal/h) & 6,014 & 5,647 \\
Hydrotreating catalyst (lb/h) & 14 & 16 \\
Hydrocracking catalyst (Ib/h) & 2 & 4
\end{tabular}




\begin{tabular}{|c|c|c|}
\hline Resource Consumption at Biorefinery & Flow rate (lb/h) & Flow rate $(\mathrm{Ib} / \mathrm{h})$ \\
\hline Blended woody biomass (wet) & 204,131 & 204,131 \\
\hline Blended woody biomass (dry) & 183,718 & 183,718 \\
\hline Sand makeup & 158 & 159 \\
\hline Natural gas & 357 & 103 \\
\hline Zeolite catalyst & $0.0 \mathrm{E}+00$ & $0.0 \mathrm{E}+00$ \\
\hline Fixed-bed CFP catalyst $\left(\mathrm{Pt} / \mathrm{TiO}_{2}\right)$ & 7 & 8 \\
\hline $\begin{array}{l}\text { Hydrotreating catalyst (sulfided CoMo or NiMo) } \\
\text { Hydrocracking catalyst (crystalline Si-Al with rare } \\
\text { earth metals) }\end{array}$ & - & - \\
\hline $\mathrm{ZnO}$ (reforming cleanup) & $9.1 \mathrm{E}-02$ & 2.6E-02 \\
\hline Hydrodesulfurization (reforming cleanup) & $3.9 \mathrm{E}-02$ & 1.1E-02 \\
\hline Steam reforming catalyst & 2.0E-01 & $5.8 \mathrm{E}-02$ \\
\hline Shift catalyst & 2.7E-01 & 7.9E-02 \\
\hline Pressure swing adsorbent & $6.9 \mathrm{E}+00$ & $2.0 \mathrm{E}+00$ \\
\hline Solvent 1 & - & 111 \\
\hline Solvent 2 & - & 3 \\
\hline 50 wt $\%$ caustic & 291 & 291 \\
\hline Net water makeup & 53,545 & 53,010 \\
\hline Boiler feedwater chemicals & 2 & 2 \\
\hline Cooling tower chemicals & 1 & 1 \\
\hline No. 2 diesel fuel & 71 & 71 \\
\hline Waste Streams & $\mathrm{lb} / \mathrm{h}$ & $\mathrm{lb} / \mathrm{h}$ \\
\hline Solids purge from fluidized bed reactors & 3,741 & 3,743 \\
\hline Wastewater & 22,147 & 24,434 \\
\hline Air Emissions & $\mathrm{lb} / \mathrm{h}$ & $\mathrm{lb} / \mathrm{h}$ \\
\hline $\mathrm{CO}_{2}$ (fossil) & 980 & 283 \\
\hline $\mathrm{CO}_{2}$ (biogenic) & 197,545 & 210,747 \\
\hline $\mathrm{CH}_{4}$ & $0.0 \mathrm{E}+00$ & $0.0 \mathrm{E}+00$ \\
\hline $\mathrm{CO}$ & $0.0 \mathrm{E}+00$ & $0.0 \mathrm{E}+00$ \\
\hline $\mathrm{NO}_{2}$ & 11 & 24 \\
\hline $\mathrm{SO}_{2}$ & 106 & 106 \\
\hline $\mathrm{H}_{2} \mathrm{O}$ & 150,488 & 142,066 \\
\hline $\mathrm{H}_{2} \mathrm{~S}$ & $0.0 \mathrm{E}+00$ & $0.0 \mathrm{E}+00$ \\
\hline \multicolumn{3}{|l|}{ Combustor Feed Stream Heating Values } \\
\hline Char combustor \% biogenic carbon & $100 \%$ & $100 \%$ \\
\hline Char combustor LHV (MMBtu/h) & 295.94 & 238.27 \\
\hline Reformer fuel combustor \% biogenic carbon & $99.17 \%$ & $99.81 \%$ \\
\hline Reformer fuel combustor LHV (MMBtu/h) & 458.66 & 571.00 \\
\hline
\end{tabular}


Table 11 shows efficiency indicators and water usage metrics in the conversion process. Carbon and energy efficiencies also reflect the sustainability of the process; conversion of biomass feedstock to desirable products benefits both the economics and sustainability. The process does not require natural gas at the biorefinery, and the model predicts that there will be a net electricity export after in-process consumption (the electricity is produced from excess process heat). However, natural gas and electricity are consumed at the petroleum refinery for cohydroprocessing, as shown in Table 10.

Table 11. Sustainability and Process Efficiency Metrics for the Conversion Process, Including CoHydroprocessing at a Petroleum Refinery

\begin{tabular}{|c|c|c|c|c|}
\hline \multirow{2}{*}{$\begin{array}{l}\text { Model } \\
\text { Electricity and coproduct credit }\end{array}$} & \multicolumn{2}{|c|}{2020 Target } & \multicolumn{2}{|c|}{2020 SOT } \\
\hline & Yes & No & Yes & No \\
\hline GHG emissions ( $\left.\mathrm{g} \mathrm{CO}_{2} \mathrm{e} / \mathrm{MJ}\right)^{a}$ & $-4.5^{b}$ & $29.3^{b}$ & $-5.2^{b}$ & $29.5^{b}$ \\
\hline Net fossil energy consumption (MJ/MJ) a & 0.02 & 0.45 & 0.01 & 0.47 \\
\hline Fuel yield (\% w/w of dry biomass) & \multicolumn{2}{|c|}{19.1} & \multicolumn{2}{|c|}{19.6} \\
\hline Fuel + coproducts yield ( $\%$ w/w of dry biomass) & \multicolumn{2}{|c|}{22.5} & \multicolumn{2}{|c|}{23.0} \\
\hline Carbon efficiency to fuels ( $\%$ of $\mathrm{C}$ in biomass) & \multicolumn{2}{|c|}{32.9} & \multicolumn{2}{|c|}{33.7} \\
\hline $\begin{array}{l}\text { Carbon efficiency to fuels and coproducts (\% C } \\
\text { of } \mathrm{C} \text { in biomass) }\end{array}$ & \multicolumn{2}{|c|}{37.2} & \multicolumn{2}{|c|}{38.0} \\
\hline Overall energy efficiency to fuels (\% LHV basis) & \multicolumn{2}{|c|}{43.4} & \multicolumn{2}{|c|}{45.1} \\
\hline Water consumption (gal/GGE fuel) & \multicolumn{2}{|c|}{$1.2^{c}$} & \multicolumn{2}{|c|}{$1.1^{c}$} \\
\hline Total fuel yield (GGE/dry U.S. ton) & \multicolumn{2}{|c|}{59.5} & \multicolumn{2}{|c|}{61.4} \\
\hline Electricity production (kWh/GGE) & \multicolumn{2}{|c|}{10.8} & \multicolumn{2}{|c|}{10.6} \\
\hline Electricity consumption (kWh/GGE) & \multicolumn{2}{|c|}{7.1} & \multicolumn{2}{|c|}{$5.9^{d}$} \\
\hline Wastewater generation (gal/GGE) & \multicolumn{2}{|c|}{0.49} & \multicolumn{2}{|c|}{0.52} \\
\hline \multicolumn{5}{|c|}{$\begin{array}{l}\text { a Calculated by ANL using Greenhouse gases, Regulated Emissions, and Energy use in Transportation v. } \\
2018 \text { [25] at the conversion step (i.e., at the biorefinery or "gate-to-gate"), excluding upstream and } \\
\text { downstream processes in the supply chain. The full SCSA results will be reported separately by ANL. }\end{array}$} \\
\hline \multicolumn{5}{|c|}{${ }^{\mathrm{b}}$ Includes emissions at petroleum refinery } \\
\hline \multicolumn{5}{|l|}{${ }^{\mathrm{c}}$ At biorefinery } \\
\hline \multicolumn{5}{|c|}{$\begin{array}{l}{ }^{\mathrm{d}} \text { Reduction in electricity consumption compared to the } 2020 \text { target is primarily due to the removal of } \\
\text { contingencies for energy consumption in the } 2020 \text { SOT model for light oxygenates purification, because a } \\
\text { more detailed model with better estimates is now available. Electricity production benefited from the } \\
\text { inclusion of steam generation from coke burnoff during CFP catalyst regeneration (not previously included), } \\
\text { and there was some reduced electricity consumption because of less char production and related burnoff } \\
\text { air-compression requirements. }\end{array}$} \\
\hline
\end{tabular}

An SCSA for the ex situ CFP pathway was conducted using ANL's Greenhouse gases, Regulated Emissions, and Energy use in Transportation (GREET) model [27]. The SCSA incorporated the feedstock modeled by INL for the 2020 SOT (50\% forest residues and 50\% clean pine) [13]. For the conversion step, when the displacement credit of coproduced electricity is excluded, fossil energy consumption is approximately $0.47 \mathrm{MJ} / \mathrm{MJ}$ for the $2020 \mathrm{SOT}$; GHG emission intensity is approximately $29.5 \mathrm{~g} \mathrm{CO}_{2} \mathrm{e} / \mathrm{MJ}$. When the displacement credit of coproduced electricity and coproducts is included, net fossil energy consumption is approximately $0.01 \mathrm{MJ} / \mathrm{MJ}$ and net GHG emission intensity is approximately $-5.2 \mathrm{~g} \mathrm{CO}_{2} \mathrm{e} / \mathrm{MJ}$. The energy self-sufficient conversion process contributes to the low fossil energy consumption and low GHG emission intensities of the ex situ CFP conversion technology at the biorefinery. The acetone and MEK coproducts provide further GHG benefits based on the life cycle analysis product displacement method [28]. 
However, there is natural gas and grid electricity consumption at the petroleum refinery that increases GHG emissions for the production of final fuel after co-hydroprocessing at the petroleum refinery.

ANL's SCSA shows that the overall modeled GHG reduction relative to a petroleum-derived gasoline baseline ( $92.7 \mathrm{~g} \mathrm{CO}_{2} \mathrm{e} / \mathrm{MJ}$ ) is $78 \%$ for the 2020 SOT base case [26]. Feedstock choices and related preprocessing, as well as refinery co-hydroprocessing, have significant impacts on the SCSA results and can thus be areas of improvement in this respect.

\section{Conclusion}

The research behind this 2020 SOT assessment helped make significant advancements toward the resolution of key uncertainties in the areas of co-hydroprocessing and the separation and purification of light oxygenated coproducts. Overall, the modeled 2020 SOT MFSP of $\$ 2.83 / \mathrm{GGE}$ with a $78 \%$ GHG reduction shows that we met the key research and associated modeled cost reduction goals we had targeted to achieve by 2022 at the onset of this project in 2014 (when we started with a modeled MFSP of \$6.27/GGE). Note that these modeled costs are based on bench-scale experiments, and challenges and risks remain to be addressed during potential future scale-up. Risks, future research, and opportunities for further improvements will be detailed in a closeout report expected to be published at the end of 2021. The modeled MFSP of $\$ 2.83 / \mathrm{GGE}$ for the 2020 SOT base case includes contingencies for some of the known uncertainties and risks. 


\section{References}

1. Dutta, A., A. Sahir, E. Tan, D. Humbird, L. Snowden-Swan, P. Meyer, J. Ross, D. Sexton, R. Yap, and J. Lukas. 2015. Process Design and Economics for the Conversion of Lignocellulosic Biomass to Hydrocarbon Fuels-Thermochemical Research Pathways with In Situ and Ex Situ Upgrading of Fast Pyrolysis Vapors. Golden, CO: National Renewable Energy Laboratory. NREL/TP-5100-62455, PNNL-23823. https://www.nrel.gov/docs/fy15osti/62455.pdf.

2. U.S. Department of Energy Office of Energy Efficiency and Renewable Energy. 2016. Bioenergy Technologies Office Multi-Year Program Plan. Washington, D.C.: U.S. Department of Energy. DOE/EE-1385. https://www.energy.gov/sites/default/files/2016/07/f33/mypp_march2016.pdf.

3. Lappas, A.A., M.C. Samolada, D.K. Iatridis, S.S. Voutetakis, and I.A. Vasalos. 2002. "Biomass pyrolysis in a circulating fluid bed reactor for the production of fuels and chemicals." Fuel 81 (16): 2087-2095. https://doi.org/10.1016/S0016-2361(02)00195-3.

4. Wan, S., T. Pham, S. Zhang, L. Lobban, D. Resasco, and R. Mallinson. 2013. "Direct catalytic upgrading of biomass pyrolysis vapors by a dual function $\mathrm{Ru} / \mathrm{TiO}_{2}$ catalyst." AIChE Journal 59 (7): 2275-2285. https://doi.org/10.1002/aic.14038.

5. Wang, K., D.C. Dayton, J.E. Peters, and O.D. Mante. 2017. "Reactive catalytic fast pyrolysis of biomass to produce high-quality bio-crude." Green Chemistry 19: 243-3251. https://doi.org/10.1039/C7GC01088E.

6. Dutta, A., J. Schaidle, D. Humbird, F. Baddour, and A. Sahir. 2016. "Conceptual Process Design and Techno-Economic Assessment of Ex Situ Catalytic Fast Pyrolysis of Biomass: A Fixed Bed Reactor Implementation Scenario for Future Feasibility." Topics in Catalysis 59 (1): 2-18. https://doi.org/10.1007/s11244-015-0500-Z.

7. Griffin, M.B., K. Iisa, H. Wang, A. Dutta, K.A. Orton, R.J. French, D.M. Santosa, et al. 2018. "Driving towards cost-competitive biofuels through catalytic fast pyrolysis by rethinking catalyst selection and reactor configuration." Energy \& Environmental Science 11: 2904-2918. http://dx.doi.org/10.1039/C8EE01872C.

8. Dutta, A., K. Iisa, M. Talmadge, C. Mukarakate, M. Griffin, E. Tan, N. Wilson, et al. 2020. Ex Situ Catalytic Fast Pyrolysis of Lignocellulosic Biomass to Hydrocarbon Fuels: 2019 State of Technology and Future Research. Golden, CO: National Renewable Energy Laboratory. NREL/TP-5100-76269. https://www.nrel.gov/docs/fy20osti/76269.pdf.

9. Internal Revenue Service. 2009. "How to Depreciate Property." Internal Revenue Service Publication 946. Washington, D.C.: United States Department of the Treasury.

10. U.S. Bureau of Labor Statistics. 2018. "PPI industry sub-sector data for Chemical Manufacturing." Accessed October 26, 2018. https://data.bls.gov/timeseries/PCU325--$\underline{325---? a m p \% 253 \text { bdata tool=XGtable\&output view=data\&include graphs=true. }}$. 
11. Chemical Engineering. 2018. "Chemical Engineering Plant Cost Index.” Accessed September 2018.

12. Aspen Plus. n.d. “Aspen Plus ${ }^{\circledR}$ V10.” Bedford, MA: AspenTech. https://www.aspentech.com.

13. Hartley, Damon S., David N. Thompson, and Hao Cai. 2021. Woody Feedstocks 2020 State of Technology Report. Idaho Falls, ID: Idaho National Laboratory. INL/EXT-2059976. https://doi.org/10.2172/1782211.

14. U.S. Department of Energy Office of Energy Efficiency and Renewable Energy. 2021. "About the Feedstock-Conversion Interface Consortium." Accessed June 2021. https://www.energy.gov/eere/bioenergy/about-feedstock-conversion-interfaceconsortium.

15. Carpenter, Daniel and Jim Parks. 2021. "FCIC Task 6: High Temperature Conversion." Presented at the DOE Bioenergy Technologies Office (BETO) 2021 Project Peer Review, March 15, 2021. https://www.energy.gov/sites/default/files/2021-04/beto-06-peerreview-2021-fcic-carpenter.pdf.

16. Baddour, F. and L. Snowden-Swan. 2017. "Catalyst Cost Model Development." Presented at the Bioenergy Technologies Office 2017 Project Peer Review, Denver, CO, March 7, 2017. https://www.energy.gov/sites/prod/files/2017/05/f34/ thermochem baddour_2.5.4.301-302.pdf.

17. U.S. Department of Energy. 2021. "Chemical Catalysis for Bioenergy Consortium." Accessed June 2021. https://www.chemcatbio.org.

18. Humbird, D., R. Davis, L. Tao, C. Kinchin, D. Hsu, A. Aden, P. Schoen, et al. 2011. Process Design and Economics for Biochemical Conversion of Lignocellulosic Biomass to Ethanol: Dilute-Acid Pretreatment and Enzymatic Hydrolysis of Corn Stover. Golden, CO: National Renewable Energy Laboratory. NREL/TP-5100-47764. https://www.nrel.gov/docs/fy11osti/47764.pdf.

19. U.S. Geological Survey. 2014. "Sand and Gravel (Industrial)." Reston, VA: U.S. Geological Survey. http://minerals.usgs.gov/minerals/pubs/commodity/silica/mcs-2014sandi.pdf.

20. Suresh, B., R. Gubler, Y. Yamaguchi, and X. He. 2013. "Hydrogen.” In Chemical Economics Handbook. Englewood, CO: IHS Chemical.

21. Peters, M.S., K.D. Timmerhaus, and R. West. 2004. Plant Design and Economics for Chemical Engineers, International Edition. 5th edition. New York: McGraw-Hill, Inc.

22. U.S. Energy Information Administration. 2011. "Annual Energy Outlook 2011. Data Table A3 - Energy Prices by Sector and Source.” Washington, D.C.: U.S. Energy Information Administration. 
23. Perry, R.H., D.W. Green, and J.O. Maloney. 1997. Perry's Chemical Engineers' Handbook. 7th edition. New York: McGraw-Hill.

24. U.S. Bureau of Labor Statistics. 2018. "Databases, Tables \& Calculators by Subject." Accessed 2018. http://data.bls.gov/cgi-bin/srgate (enter CEU3232500008 series id).

25. Argonne National Laboratory. 2014. "GREET 2016 Model." https://greet.es.anl.gov/publication-greet-model.

26. Cai, H., L. Ou, M. Wang, R. Davis, A. Dutta, K. Harris, M. Wiatrowski, et al. 2021. Supply Chain Sustainability Analysis of Renewable Hydrocarbon Fuels via Indirect Liquefaction, Ex Situ Catalytic Fast Pyrolysis, Hydrothermal Liquefaction, Combined Algal Processing, and Biochemical Conversion: Update of the 2020 State-of-Technology Cases. Lemont, IL: Argonne National Laboratory. ANL/ESD-21/1.

27. Wang, Michael, Amgad Elgowainy, Pahola Thathiana Benavides, Andrew Burnham, Hao Cai, Qiang Dai, Troy Robert Hawkins, et al. 2018. Summary of Expansions and Updates in GREET 2018. Lemont, IL: Argonne National Laboratory. ANL-18-38.

28. Cai, H., J. Han, M. Wang, R. Davis, M. Biddy, and E. Tan. 2018. "Life-cycle analysis of integrated biorefineries with co-production of biofuels and bio-based chemicals: coproduct handling methods and implications." Biofuels, Bioproducts and Biorefining 12: 815-833. https://doi.org/10.1002/bbb.1893. 


\section{Appendix A: 2014-2020 SOTs}

\section{Table A-1. Processing Area Cost Contributions and Key Technical Parameters}

\begin{tabular}{|c|c|c|c|c|c|c|c|c|}
\hline $\begin{array}{l}\text { Processing Area Cost Contributions \& Key Technical } \\
\text { Parameters }\end{array}$ & Units & 2014 SOT & 2015 SOT & 2016 SOT & 2017 SOT $^{\mu}$ & 2018 SOT & 2019 SOT & 2020 SOT \\
\hline $\begin{array}{l}\text { Process Concept: Hydrocarbon Fuel Production } \\
\text { via Ex Situ Upgrading of Fast Pyrolysis Vapors }\end{array}$ & & Clean Pine & Clean Pine & Clean Pine & Clean Pine & Clean Pine & $\begin{array}{l}\text { 50\%Residue } \\
\text { s/50\% }\end{array}$ & $\begin{array}{l}\text { 50\%Residue } \\
\text { s/50\%Pine }\end{array}$ \\
\hline Year \$ Basis & & 2016 & 2016 & 2016 & 2016 & 2016 & 2016 & 2016 \\
\hline Projected Minimum Fuel Selling Price ${ }^{\star}$ & S/GGE* & $\$ 6.27$ & $\$ 5.44$ & $\$ 4.90$ & $\$ 4.09$ & $\$ 3.80$ & $\$ 3.33$ & $\$ 2.83$ \\
\hline Conversion Contribution & S/GGE* & $\$ 3.66$ & $\$ 3.30$ & $\$ 3.08$ & $\$ 2.82$ & $\$ 2.44$ & $\$ 2.14$ & $\$ 1.74$ \\
\hline Total Project Investment per Annual GGE & S/GGE-yr & $\$ 18.50$ & $\$ 16.46$ & $\$ 14.94$ & $\$ 12.17$ & $\$ 12.47$ & $\$ 13.53$ & $\$ 11.64$ \\
\hline Plant Capacity (Dry Feedstock Basis) & metric tons/day & 2,000 & 2,000 & 2,000 & 2,000 & 2,000 & 2,000 & 2,000 \\
\hline Total Gasoline Equivalent Yield & GGE/dry US ton & 42 & 46 & 51 & 69 & 65 & 59 & 61 \\
\hline Diesel-Range Product Proportion (GGE* basis) & $\%$ of fuel product & $15 \%$ & $15 \%$ & $15 \%$ & $52 \%$ & $52 \%$ & $48 \%$ & $50 \%$ \\
\hline \multicolumn{9}{|l|}{ Feedstock } \\
\hline Total Cost Contribution" & S/GGE & $\$ 2.60$ & $\$ 2.14$ & $\$ 1.82$ & $\$ 1.27$ & $\$ 1.36$ & $\$ 1.18$ & $\$ 1.10$ \\
\hline Capital Cost Contribution" & \$/GGE & $\$ 0.00$ & $\$ 0.00$ & $\$ 0.00$ & $\$ 0.00$ & $\$ 0.00$ & $\$ 0.00$ & $\$ 0.00$ \\
\hline Operating Cost Contribution" & \$/GGE & $\$ 2.60$ & $\$ 2.14$ & $\$ 1.81$ & $\$ 1.27$ & $\$ 1.35$ & $\$ 1.18$ & $\$ 1.09$ \\
\hline Feedstock Cost" & S/ Dry US Ton & $\$ 109.01$ & $\$ 98.31$ & $\$ 92.70$ & $\$ 87.82$ & $\$ 87.82$ & $\$ 70.15$ & $\$ 67.03$ \\
\hline Feedstock Moisture at Plant Gate & Wt \% $\mathrm{H}_{2} \mathrm{O}$ & $10 \%$ & $10 \%$ & $10 \%$ & $10 \%$ & $10 \%$ & $10 \%$ & $10 \%$ \\
\hline Feed Moisture Content to Pyrolyzer & wt $\% \mathrm{H}_{2} \mathrm{O}$ & $10 \%$ & $10 \%$ & $10 \%$ & $10 \%$ & $10 \%$ & $10 \%$ & $10 \%$ \\
\hline Energy Content (LHV, Dry Basis) & $\mathrm{BTU} / \mathrm{lb}$ & 8,000 & 8,000 & 8,000 & 8,000 & 8,000 & 7,900 & 7,900 \\
\hline \multicolumn{9}{|l|}{ Pyrolysis and Vapor Upgrading } \\
\hline Total Cost Contribution & S/GGE & $\$ 2.34$ & $\$ 2.03$ & $\$ 1.84$ & $\$ 1.46$ & $\$ 1.10$ & $\$ 1.14$ & $\$ 1.07$ \\
\hline Capital Cost Contribution & \$/GGE & $\$ 0.95$ & $\$ 0.82$ & $\$ 0.74$ & $\$ 0.65$ & $\$ 0.60$ & $\$ 0.63$ & $\$ 0.58$ \\
\hline Operating Cost Contribution & \$/GGE & $\$ 1.39$ & $\$ 1.21$ & $\$ 1.09$ & $\$ 0.80$ & $\$ 0.50$ & $\$ 0.51$ & $\$ 0.49$ \\
\hline Ex Situ Reactor Configuration & reactor type & Fluidized Bed & Fluidized Bed & Fluidized Bed & Fixed Bed & Fixed Bed & Fixed Bed & Fixed Bed \\
\hline Ratio of Online:Regenerating Fixed Bed Reactors & ratio & N/A & N/A & N/A & $2: 5$ & $2: 3$ & $2: 2$ & $2: 2$ \\
\hline Gas Phase & wt $\%$ of dry biomass & $35 \%$ & $36 \%$ & $34 \%$ & $31 \%$ & $35 \%$ & $38 \%$ & $42 \%$ \\
\hline Aqueous Phase & wt $\%$ of dry biomass & $25 \%$ & $25 \%$ & $24 \%$ & $27 \%$ & $22 \%$ & $24 \%$ & $20 \%$ \\
\hline Carbon Loss & $\%$ of $\mathrm{C}$ in biomass & $2.9 \%$ & $2.9 \%$ & $3.4 \%$ & $2.9 \%$ & $5.0 \%$ & $4.4 \%$ & $1.8 \%$ \\
\hline Organic Phase & wt $\%$ of dry biomass & $17.5 \%$ & $18.6 \%$ & $21.8 \%$ & $28.3 \%$ & $27.9 \%$ & $23.2 \%$ & $24.0 \%$ \\
\hline H/C Molar Ratio & ratio & 1.1 & 1.1 & 1.1 & 1.2 & 1.2 & 1.2 & 1.3 \\
\hline Oxygen & wt $\%$ of organic phase & $15.0 \%$ & $13.3 \%$ & $16.8 \%$ & $16.5 \%$ & $18.6 \%$ & $15.1 \%$ & $16.6 \%$ \\
\hline Carbon Efficiency & $\%$ of $\mathrm{C}$ in biomass & $27 \%$ & $29 \%$ & $33 \%$ & $42 \%$ & $40 \%$ & $35 \%$ & $36 \%$ \\
\hline Solid Losses (Char + Coke) & wt $\%$ of dry biomass & $23 \%$ & $21 \%$ & $20 \%$ & $14 \%$ & $15 \%$ & $14 \%$ & $13 \%$ \\
\hline Char & wt $\%$ of dry biomass & $12.0 \%$ & $11.0 \%$ & $12.0 \%$ & $10.4 \%$ & $11.7 \%$ & $11.6 \%$ & $11.1 \%$ \\
\hline Coke & wt $\%$ of dry biomass & $11.0 \%$ & $9.5 \%$ & $8.3 \%$ & $3.3 \%$ & $3.7 \%$ & $2.3 \%$ & $1.7 \%$ \\
\hline \multicolumn{9}{|l|}{ Vapor Quench, Co-Product Recovery } \\
\hline Total Cost Contribution & \$/GGE & $\$ 0.35$ & $\$ 0.33$ & $\$ 0.28$ & $\$ 0.20$ & $\$ 0.22$ & $\$ 0.34$ & $\$ 0.45$ \\
\hline Capital Cost Contribution & \$/GGE & $\$ 0.20$ & $\$ 0.19$ & $\$ 0.16$ & $\$ 0.12$ & $\$ 0.13$ & $\$ 0.22$ & $\$ 0.28$ \\
\hline Operating Cost Contribution & \$/GGE & $\$ 0.15$ & $\$ 0.14$ & $\$ 0.12$ & $\$ 0.08$ & $\$ 0.09$ & $\$ 0.12$ & \$0.18 \\
\hline
\end{tabular}

(continued next page) 
(continued from previous page)

\begin{tabular}{|c|c|c|c|c|c|c|c|c|}
\hline \multicolumn{2}{|l|}{ Hydroprocessing \& Separation / Refinery Co-Processing } & \multirow{2}{*}{$\frac{2014 \text { SOT }}{\text { S0.33 }}$} & \multirow{2}{*}{$\begin{aligned} 2015 \text { SOT } \\
\text { S0.31 }\end{aligned}$} & \multirow{2}{*}{$\begin{array}{c}2016 \text { SOT } \\
\text { s0.34 }\end{array}$} & \multirow{2}{*}{\begin{tabular}{|c|}
2017 SOTJI \\
S0.35
\end{tabular}} & \multirow{2}{*}{2018 SOT } & \multirow{2}{*}{$\frac{2019 \text { SOT }}{\text { S0.30 }}$} & \multirow{2}{*}{$\frac{2020 \text { SOT }}{50.23}$} \\
\hline Total Cost Contribution & S/GGE & & & & & & & \\
\hline Capital Cost Contribution & S/GGE & \$0.17 & & & & & & \\
\hline Operating Cost Contribution & S/GGE & $\$ 0.15$ & \$0.14 & $\$ 0.16$ & 50.16 & \$0.18 & \$0.14 & 50.23 \\
\hline Carbon Efficiency of Organic Liquid Feed to Fuels & $\%$ & $88.4 \%$ & $89.5 \%$ & $87.2 \%$ & $91.0 \%$ & $89.0 \%$ & $93.5 \%$ & $94.5 \%$ \\
\hline Hydrotreating Pressure & psia & 2,000 & 2,000 & 2,000 & 1,900 & 1,900 & 1,900 & 1,900 \\
\hline Oxygen Content in Cumulative Fuel Product & wt \% & $0.8 \%$ & $0.8 \%$ & $0.8 \%$ & $0.6 \%$ & $0.5 \%$ & $0.5 \%$ & $0.5 \%$ \\
\hline \multicolumn{9}{|l|}{ Hydrogen Production } \\
\hline $\begin{array}{l}\text { Total Cost Contribution } \\
\end{array}$ & S/GGE & $\$ 0.61$ & $\$ 0.56$ & $\$ 0.60$ & 50.62 & $\$ 0.51$ & 50.61 & 50.44 \\
\hline Capital Cost Contribution & S/GGE & $\$ 0.39$ & $\$ 0.36$ & $\$ 0.38$ & 50.41 & 50.33 & $\$ 0.39$ & $\$ 0.28$ \\
\hline Operating Cost Contribution & S/GGE & $\$ 0.22$ & 90.20 & $\$ 0.22$ & $\$ 0.21$ & 50.18 & 90.22 & 50.16 \\
\hline Additional Natural Gas (NG) at the Biorefinerytz & $\%$ of biomass LHV & $0.3 \%$ & $0.1 \%$ & $0.2 \%$ & $0.1 \%$ & $0.3 \%$ & $0.1 \%$ & $0.1 \%$ \\
\hline \multicolumn{9}{|l|}{ CoProducts } \\
\hline Total Cost Contribution & S/GGE & & & & & & $(\$ 0.52)$ & $(\$ 0.55)$ \\
\hline Capital Cost Contribution ${ }^{*}$ & S/GGE & & & & & & & \\
\hline Operating Cost Contribution ${ }^{*}$ & S/GGE & & & & & & & \\
\hline CoProduct Credit & S/GGE* & & & & & & $(50.52)$ & $(\$ 0.55)$ \\
\hline \multicolumn{9}{|l|}{ Balance of Plant } \\
\hline Total Cost Contribution & S/GGE & 50.04 & S0.07 & 50.03 & 50.20 & 50.23 & \$0.27 & 50.09 \\
\hline Capital Cost Contribution & S/GGE & 50.80 & 50.71 & 50.56 & 50.43 & 50.46 & 50.45 & 50.46 \\
\hline Operating Cost Contribution & S/GGE & $(\$ 0.76)$ & $(\$ 0.64)$ & $(\$ 0.54)$ & $(\$ 0.23)$ & $(50.23)$ & $(50.18)$ & $(\$ 0.37)$ \\
\hline $\begin{array}{l}\text { Electricity Production from Steam Turbine (credit included in op. } \\
\text { cost above) }\end{array}$ & S/GGE* & $(\$ 1.12)$ & $(\$ 0.96)$ & $(\$ 0.78)$ & $(\$ 0.42)$ & $(\$ 0.45)$ & $(\$ 0.40)$ & $(\$ 0.57)$ \\
\hline \multicolumn{9}{|l|}{ Sustainability and Process Efficiency Metrics } \\
\hline Fuel and Coproducts Yield by Weight of Biomass & $\%$ w/w of dry biomass & $13.7 \%$ & $15.0 \%$ & $16.5 \%$ & $22.2 \%$ & $20.9 \%$ & $22.5 \%$ & $23.0 \%$ \\
\hline Carbon Efficiency of Biomass to Fuels and Coproducts & $\% \mathrm{C}$ in Feedstock & $23.5 \%$ & $25.9 \%$ & $28.3 \%$ & $38.1 \%$ & $35.9 \%$ & $37.2 \%$ & $38.0 \%$ \\
\hline Overall Carbon Efficiency to Liquid Hydrocarbon Fuels & $\% \mathrm{C}$ in Feedstock & $23.5 \%$ & $25.9 \%$ & $28.3 \%$ & $38.1 \%$ & $35.9 \%$ & $33.0 \%$ & $33.7 \%$ \\
\hline Overall Energy Efficiency to Liquid Hydrocarbon Fuels & \% LHV of Feedstock & $30.5 \%$ & $33.4 \%$ & $37.1 \%$ & $50.3 \%$ & $47.2 \%$ & $43.6 \%$ & $45.1 \%$ \\
\hline Electricity Production & kWh/GGE & 21.0 & 18.0 & 14.7 & 8.0 & 8.7 & 7.8 & 10.6 \\
\hline Electricity Consumption (Entire Process) & kWh/GGE & 12.7 & 11.0 & 9.6 & 6.4 & 7.5 & 7.4 & 5.9 \\
\hline Water Consumption in Conversion Process & $\mathrm{gal} \mathrm{H}_{2} \mathrm{O} / \mathrm{GGE}$ & 1.4 & 1.4 & 1.3 & 1.5 & 1.4 & 1.7 & 1.1 \\
\hline TEA Reference File & & $\begin{array}{l}\text { PyVPU- } \\
\text { v2189 ES- } \\
\text { Fludidized Bed } \\
\text { - Fr14 SOT } \\
\text { (20165)- } \\
\text { v02d-feed- } \\
\text { 109.01.xlsm }\end{array}$ & \begin{tabular}{|c} 
PyVPU- \\
v218g ES - \\
Fluidized Bed \\
- FY15 SOT \\
(20165)- \\
v02d-feed- \\
98.31.xlsm
\end{tabular} & $\begin{array}{c}\text { PyVPU- } \\
\text { V218g ES- } \\
\text { Fluidized Bed } \\
\text { - FY16 SOT } \\
\text { (20165)- } \\
\text { V02d-feed- } \\
\text { 92.70.xlsm }\end{array}$ & \begin{tabular}{|c|} 
PyVPU- \\
v218h ES \\
FixedBed- \\
v49-r046- \\
AP10- \\
FY17SOT \\
(2016S)- \\
V02d-b.xism
\end{tabular} & $\begin{array}{c}\text { PyVPU- } \\
\text { v218h ES } \\
\text { FixedBed- } \\
\text { v49-1046- } \\
\text { AP10- } \\
\text { FY18SOT } \\
\text { (20165)- } \\
\text { V09d- } \\
\text { Revised } \\
\text { 40.4pct C-Eff- } \\
\text { b.xlsm }\end{array}$ & \begin{tabular}{|c} 
PyVPU- \\
v218h ES \\
Fixededed- \\
v49-ro46- \\
AP10- \\
FY1950T- \\
V18-Acetone- \\
MEK- \\
\$3.33.xism
\end{tabular} & \begin{tabular}{|l} 
PyVPU-2020- \\
Vo96- \\
HT__tandalon \\
e-LO-R008- \\
copied \\
data.xlsm
\end{tabular} \\
\hline
\end{tabular}

$\Delta$ Conceptual design result. ${ }^{\Delta}$ SOT: state of technology. *Gasoline gallon equivalent (GGE) on a lower heating value (LHV) basis. ** Natural gas stream was negligible in most of the biorefinery models. This was included to maintain model flexibility to allow natural gas use as an option. ${ }^{\ddagger} 2030$ projections are based on high-level estimates and will be modeled in detail in future years. It is proposed that co-

hydroprocessing of CFP oil will occur at a petroleum refinery. Capital for hydrogen production is included, whereas natural gas feed for hydrogen production is not included because credit is not taken for an equivalent amount of fuel gas from the CFP biorefinery. Coproduct credit is based on a preliminary estimate of diverting $20 \%$ CFP oil to produce coproducts, including from the organic liquid phase. ${ }^{\circ}$ Capital and operating costs for coproduct recovery in the 2019-2022 models are included in the "Vapor Quench, Co-Product Recovery" section. ${ }^{+\dagger}$ Modeled ash is $1.75 \%$ for 2019 and 2020 , and less than $1 \%$ for all other years. " An additional biomass heater is included as a small additional in-plant cost, as shown in https://www.nrel.gov/docs/fy15osti/62455.pdf. ${ }^{1 J}$ For the 2017 SOT unquantified portion of CFP, yields were prorated to solids, liquids, and gases using measured yields. "Small adjustments made to previously published feedstock cost estimates for 2014-2016. 